\title{
Explicit Pieri Inclusions
}

\author{
Markus Hunziker \\ Department of Mathematics \\ Baylor University \\ Texas, U.S.A.
}

markus_hunziker@baylor.edu

\author{
John A. Miller \\ Department of Mathematics, Analytics \& Technology \\ Rockhurst University \\ Missouri, U.S.A. \\ john.miller@rockhurst.edu \\ Mark Sepanski \\ Department of Mathematics \\ Baylor University \\ Texas, U.S.A. \\ mark_sepanski@baylor.edu
}

Submitted: Dec 13, 2019; Accepted: Aug 2, 2021; Published: Sep 10, 2021

(C) The authors. Released under the CC BY license (International 4.0).

\begin{abstract}
By the Pieri rule, the tensor product of an exterior power and a finite-dimensional irreducible representation of a general linear group has a multiplicity-free decomposition. The embeddings of the constituents are called Pieri inclusions and were first studied by Weyman in his thesis and described explicitly by Olver. More recently, these maps have appeared in the work of Eisenbud, Fløystad, and Weyman and of Sam and Weyman to compute pure free resolutions for classical groups.

In this paper, we give a new closed form, non-recursive description of Pieri inclusions. For partitions with a bounded number of distinct parts, the resulting algorithm has polynomial time complexity whereas the previously known algorithm has exponential time complexity.
\end{abstract}

Mathematics Subject Classifications: 05E16

\section{Introduction}

\section{$1.1 \quad$ Background}

Let $\lambda=\left(\lambda_{1}, \lambda_{2}, \ldots\right)$ be a partition, i.e., a sequence of non-negative integers in weakly decreasing order. The number of nonzero parts $\lambda_{i}$ is called the length of $\lambda$, denoted by $\ell(\lambda)$. If $V$ is a complex vector space of dimension $n \leqslant \ell(\lambda)$, we can apply the Schur-Weyl functor $\mathbb{S}_{\lambda}(V)$ (as in [3]) to $V$ to obtain an irreducible representation $\mathbb{S}_{\lambda}(V)$ of the general linear group $G L(V)$. It follows from Pieri's formula for the product of an elementary symmetric 
polynomial and a Schur polynomial that the tensor product representation $\wedge^{m}(V) \otimes \mathbb{S}_{\lambda}(V)$ decomposes multiplicity-free into a direct sum of irreducible representations

$$
\wedge^{m}(V) \otimes \mathbb{S}_{\lambda}(V) \cong \bigoplus_{\mu} \mathbb{S}_{\mu}(V)
$$

where the sum is over all partitions $\mu$ with $l(\mu) \leqslant n$ whose Young diagram is obtained from the Young diagram of $\lambda$ by adding exactly $m$ boxes, at most one to each row. Since the decomposition is multiplicity-free, it is natural to ask for explicit descriptions of the embeddings $\Phi_{m}: \mathbb{S}_{\mu}(V) \hookrightarrow \wedge^{m}(V) \otimes \mathbb{S}_{\lambda}(V)$. Following [14], we call these embeddings (skew) Pieri inclusions.

Given a basis $\left\{e_{1}, e_{2}, \ldots, e_{n}\right\}$ of $V$, the representation $\mathbb{S}_{\lambda}(V)$ is equipped with a canonical basis indexed by the set of semistandard tableaux of shape $\lambda$ with fillings from the set $\{1, \ldots, n\}$. In [10], Olver gave an explicit description of the Pieri inclusions with respect to these canonical bases in the special case when $m=1$. When $m>1$, the Pieri inclusion $\Phi_{m}$ can be obtained by iteration of the special case [14, Corollary 1.8]. In [9], these Pieri inclusions are extended to the characteristic-free setting.

The main purpose of this paper is to give a new combinatorial description of $\Phi_{m}$ that (a) leads to a more efficient algorithm and (b) can be given in a general closed form (avoiding iteration) for $m \geqslant 1$. In regard to (a), we will show that our algorithm achieves an exponential speed-up over Olver's algorithm when it is restricted to partitions with a bounded number of distinct parts. More precisely, if we fix a positive integer $N$ and consider partitions $\lambda$ that can be written in exponential notation as $\lambda=\left(1^{h_{1}}, 2^{h_{2}}, 3^{h_{3}}, \ldots\right)$ with at most $N$ nonzero exponents $h_{i}$, then our algorithm to compute the image of a highest weight vector under a Pieri inclusion $\Phi_{1}: \mathbb{S}_{\mu}(V) \hookrightarrow V \otimes \mathbb{S}_{\lambda}(V)$ has a run-time complexity of $O\left(l(\lambda)^{N}\right)$, whereas Olver's algorithm has a run-time complexity of $\Omega\left(2^{\ell(\lambda)}\right)$.

\subsection{The General Pieri Inclusion}

Our general formula for a Pieri inclusion $\Phi_{m}: \mathbb{S}_{\mu}(V) \hookrightarrow \wedge^{m}(V) \otimes \mathbb{S}_{\lambda}(V)$, where $\mu / \lambda$ is a skew diagram with no two boxes in the same row, is as follows. If $T$ is a semistandard tableau of shape $\mu$ with filling in $\{1, \ldots, n\}$ and $e_{T} \in \mathbb{S}_{\mu}(V)$ is the corresponding basis element, then

$$
\Phi_{m}\left(e_{T}\right)=\sum_{P} \frac{(-1)^{P}}{H(P)} P(T)
$$

where the sum is over a certain set of " $m$-paths" $P$ which remove $m$ boxes from the shape $\mu,(-1)^{P}$ is a sign, $H(P)$ is a positive integer that is a product of certain "hook lengths." For a precise definition, see Sections 3 and 4.

We will write the path $P$ acting on $T$ as

$$
P(T)=e_{Y_{P}} \otimes e_{T_{P}}
$$

where $e_{Y_{P}}=e_{i_{1}} \wedge \cdots \wedge e_{i_{m}} \in \wedge^{m}(V)$ is given by the entries of the boxes removed by $P$, and $T_{P}$ is a (not necessarily semistandard) tableau of shape $\lambda$ with filings in $\{1, \ldots, n\}$ 
such that

\{numbers appearing in $T\}=\left\{\right.$ numbers appearing in $\left.Y_{P}\right\} \cup\left\{\right.$ numbers appearing in $\left.T_{P}\right\}$ as a multi-set. All of this will be defined rigorously in Sections 3 and 4 .

\subsection{An Example of One Box Removal Acting on a Highest Weight Vector}

To illustrate how our formula works, we look at an example in the case when $n=4$ and $m=1$. Let $\mu=(2,1,1,1)$, and $\lambda=(2,1,1)$. Then the Schur-Weyl module $\mathbb{S}_{\mu}(V)$ appears as a summand in the decomposition of $\mathbb{S}_{(1)}(V) \otimes \mathbb{S}_{(2,1,1)}(V)=V \otimes \mathbb{S}_{(2,1,1)}$,

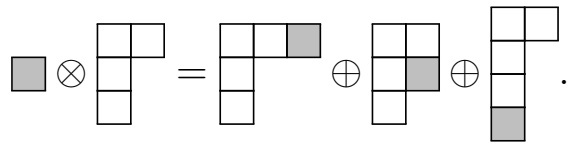

Consider the Pieri inclusion

$$
\Phi_{1}: \mathbb{S}_{(2,1,1,1)}(V) \hookrightarrow V \otimes \mathbb{S}_{(2,1,1)}(V)
$$

By abuse of notation, we will identify semistandard tableaux and their corresponding basis vectors. We will compute

$$
\Phi_{1}\left(\begin{array}{ll}
\hline & 1 \\
\hline 2 & \\
\hline 3 & 4
\end{array}\right)=\sum_{P} \frac{(-1)^{P}}{H(P)} P\left(\begin{array}{ll}
1 & 1 \\
\hline 2 & \\
\hline 3 & \\
4 &
\end{array}\right) .
$$

The sum is over all "1-paths" $P$ on $\mu$, that is, certain maps on the boxes in $\mu$ that remove a single box. Below we illustrate all such 1-paths with arrows, shading the boxes on which the path acts. We will view the box removed by a 1-path as being moved to the top of the diagram in a "zeroth row" of the diagram. We give the image up to a row permutation, so that it is semi-standard.
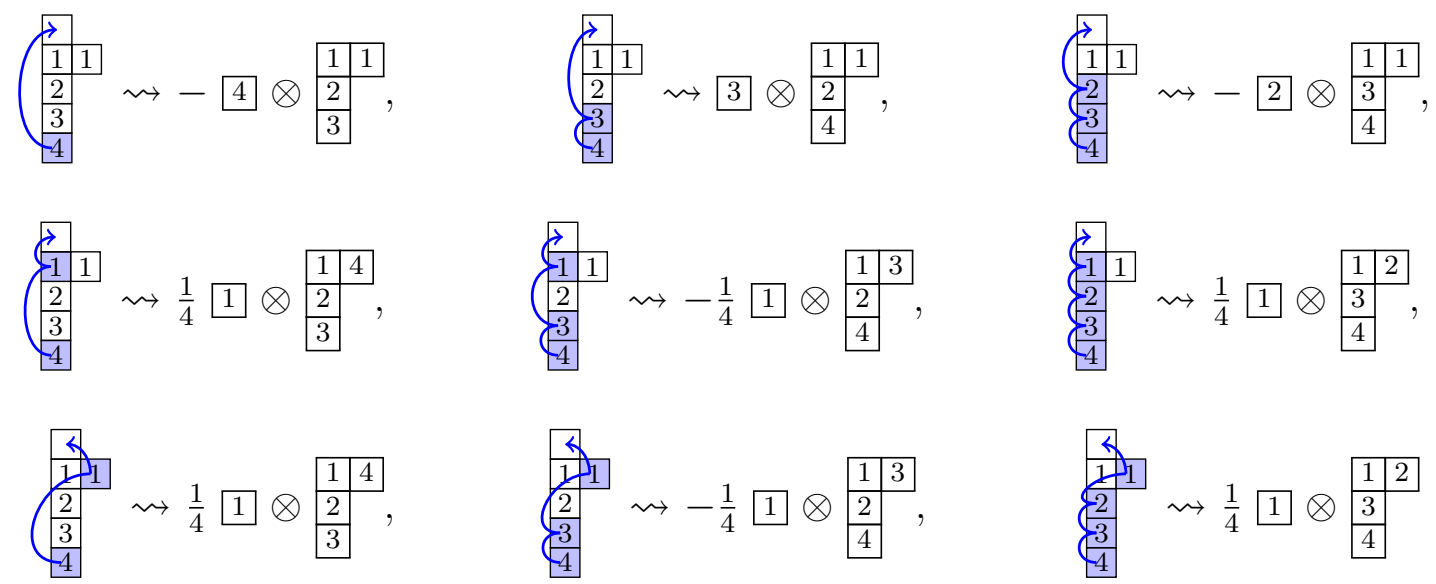
Thus, up to row permutations we have

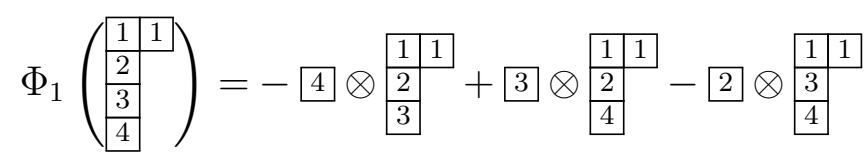

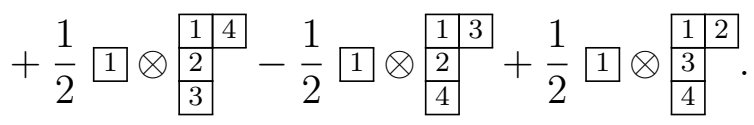

\subsection{An Example of One Box Removal Requiring Straightening}

In Section 1.3, all terms $T_{P}$ that appeared were (after row permutations) semi-standard. We now compute an example where some of the terms that appear in the image are not semi-standard, and so must be straightened. Let $\Phi_{1}$ be as in Section 1.3, we will compute

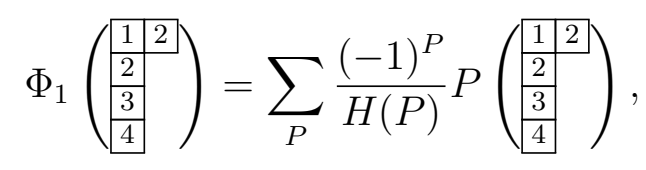

where the terms of the sum in the image are again indexed by the 1-paths on $\lambda$ removing a single box, which we illustrate below. As before, we give the image up to row permutations and we now star the terms that need to be straightened.

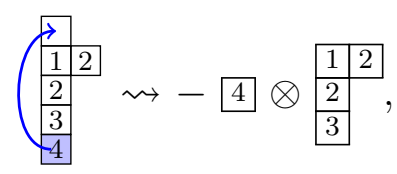

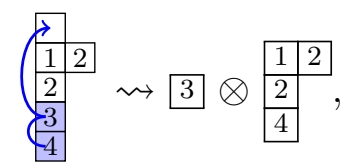

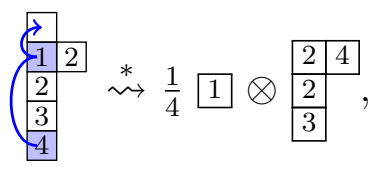

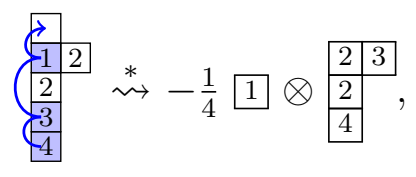

$$
\begin{aligned}
& \begin{array}{|l|l|}
\hline y_{2} \\
\hline 2 \\
\hline 3 \\
\hline 4 \\
\hline
\end{array}
\end{aligned}
$$

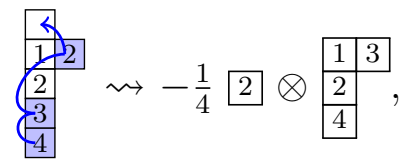
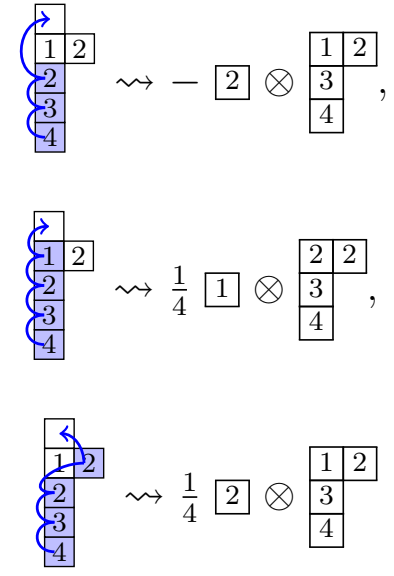

In this case, we must straighten the image of two of the 1-paths (starred), which we show in Section 3.5. After straightening we have, up to row permutations,

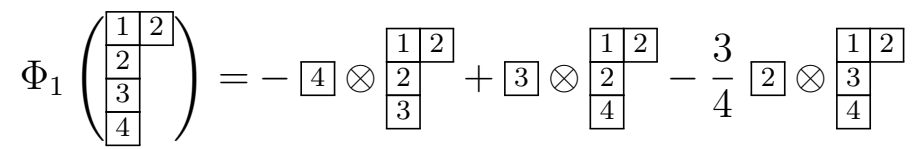

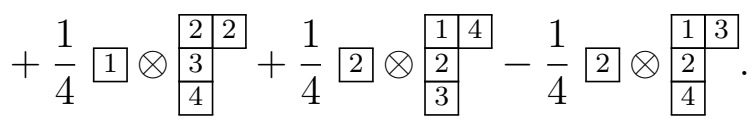

For an example of two box removal, see Section 4.4. 


\subsection{Motivation and Organization}

Part of the motivation for giving explicit descriptions of Pieri inclusions comes from the frequent use of Pieri inclusions to construct differentials of complexes and resolutions. For example, in results of Eisenbud, Fløystad, and Weyman [1] and of Sam and Weyman [14], Pieri inclusions are used to compute pure free resolutions for classical groups and, in [11], Weyman and Pragacz use Pieri inclusion maps to describe Lascoux resolutions.

In a forthcoming paper, [7], we will show how our description of Pieri inclusions can be used to explicitly describe the differentials in minimal free resolutions of modules of covariants (in the context of Weyl's fundamental theorems). These resolutions will be obtained via Howe duality from Bernstein-Gelfand-Gelfand resolutions of unitary highest weight modules, the terms of which are direct sums of (parabolic) Verma modules.

Sam has also built a package for Macaulay2 (PieriMaps, [12]) that computes Pieri inclusions explicitly using the algorithm from [14]. Once the new algorithm, with an exponential to polynomial time complexity improvement, is implemented in [12] it could benefit computational applications of PieriMaps including [2], [4], [6], and [8].

This paper is organized in the following way. In Section 2 we construct the Schur-Weyl modules $\mathbb{S}_{\lambda}(V)$. In Sections 3 and 4 we describe the construction of the Pieri inclusion in the one box removal case $(V)$ and $m$ box removal case $\left(\wedge^{m}(V)\right)$, respectively. In Sections 6 and 7 we show that the Pieri inclusions are $G L(V)$-maps in the one box removal and $m$ box removal cases, respectively, with the tools for these proofs given in Section 5 . In Section 8 we show the one box removal map is the negative of Olver's description via the uniqueness of an equivariant map and, in a similar way, show that the $m$ box removal case is equal to iterating one box removal. We also show in this section that the same description of Pieri inclusions gives a map in the case $\mathbb{S}_{\mu}(V) \hookrightarrow \operatorname{Sym}^{m}(V) \otimes \mathbb{S}_{\lambda}(V)$. Finally, in Section 9 we compare the computational complexity of the one box removal description to that of Olver.

\section{Constructing Schur-Weyl Modules}

From now on, let $\lambda=\left(\lambda_{1}, \ldots, \lambda_{r}\right)$ be a fixed partition of $d$. Let $\mathcal{T}_{\lambda, n}$ be the set of all tableau $T$ with shape $\lambda$ and arbitrary filling from the alphabet $\{1, \ldots, n\}$. A tableau $T \in \mathcal{T}_{\lambda, n}$ is called semi-standard if the filling is non-decreasing across the rows and strictly increasing down the columns. Fix the canonical tableau $T_{0}$ of shape $\lambda$ labeled with $\{1, \ldots, d\}$, starting with the top left most box and filling across each row, so the first box of the first row is labeled 1, the first box of the second row is labeled $\lambda_{1}+1$, etc. Via this labeling, the symmetric group $\mathfrak{S}_{d}$ acts on the set of tableau with shape $\lambda$ with respect to any given alphabet.

Let

$$
P=P_{\lambda}=\left\{\pi \in \mathfrak{S}_{d}: \pi \text { preserves the rows of } T_{0}\right\}
$$

and

$$
Q=Q_{\lambda}=\left\{\sigma \in \mathfrak{S}_{d}: \sigma \text { preserves the columns of } T_{0}\right\}
$$


As elements of the group algebra of $\mathfrak{S}_{d}, \mathbb{C S}_{d}$, define

$$
A_{\lambda}=\sum_{\pi \in P} \pi \quad \text { and } \quad B_{\lambda}=\sum_{\sigma \in Q}(-1)^{\sigma} \sigma .
$$

The Young Symmetrizer is then defined as $C_{\lambda}=A_{\lambda} B_{\lambda}$. Note this convention symmetrizes along rows first and antisymmetrizes along columns second (following, for example, [13]).

From now on, fix a complex vector space $V$ of dimension $n$. Let $\mathfrak{S}_{d}$ also act on elements of $V^{\otimes d}$ by permuting the factors. In particular, $C_{\lambda}$ acts on $V^{\otimes d}$. The corresponding SchurWeyl module is $\mathbb{S}_{\lambda}(V)=C_{\lambda} \cdot V^{\otimes d}$. Clearly $\mathbb{S}_{\lambda}(V)$ is a $G L(V)$-module. When the number of rows, $r$, of $\lambda$ is at most $n$, it is known that $\mathbb{S}_{\lambda}(V)$ is an irreducible representation of $G L(V)$ and that all polynomial irreducible representations are constructed this way up to isomorphism.

Write $\left\{e_{i}\right\}_{1 \leqslant i \leqslant n}$ for the standard basis of $V$. For $T \in \mathcal{T}_{\lambda, n}$, define $e_{T} \in \mathbb{S}_{\lambda}$ by

$$
e_{T}=C_{\lambda} \cdot\left(\left(e_{T_{1,1}} \otimes \cdots \otimes e_{T_{1, \lambda_{1}}}\right) \otimes \cdots \otimes\left(e_{T_{r, 1}} \otimes \cdots \otimes e_{T_{r, \lambda_{r}}}\right)\right)
$$

where $T_{i, j}$ is the entry in the $i$ th row and $j$ th column of $T$ starting from the top left. Clearly $\mathbb{S}_{\lambda}(V)$ is spanned by such elements, and it is known that a basis is given by the semistandard ones. $[5, \S 8]$

Let $V \bullet$ be the standard flag in $V$,

$$
V_{\bullet}: \quad V_{i}=\operatorname{span}\left\{e_{1}, \ldots, e_{i}\right\} \quad(1 \leqslant i \leqslant n) .
$$

Let $B \subset G L(V)$ be the Borel subgroup given by

$$
B=\left\{g \in G L(V): g V_{i} \subset V_{i} \text { for } 1 \leqslant i \leqslant n\right\} .
$$

Throughout this paper, all highest weights are with respect to $B$. The highest weight vector of $\mathbb{S}_{\lambda}(V)$ is

$$
e_{T_{\lambda}}=C_{\lambda} \cdot(\underbrace{\left(e_{1} \otimes \cdots \otimes e_{1}\right.}_{\lambda_{1}}) \otimes \cdots \otimes \underbrace{\left(e_{r} \otimes \cdots \otimes e_{r}\right.}_{\lambda_{r}})) .
$$

That is, $T_{\lambda}$ is the tableau of shape $\lambda$ with all ones in the first row, all twos in the second row, etc. For example, if $\lambda=(5,3,3,1,1)$,

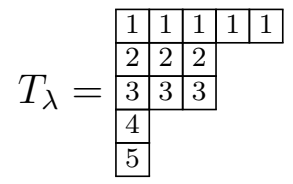

and

$$
e_{T_{\lambda}}=C_{\lambda} \cdot\left(e_{1} \otimes e_{1} \otimes e_{1} \otimes e_{1} \otimes e_{1} \otimes e_{2} \otimes e_{2} \otimes e_{2} \otimes e_{3} \otimes e_{3} \otimes e_{3} \otimes e_{4} \otimes e_{5}\right) .
$$

For any subset $A$ of boxes of $T_{0}$, let $w_{A}$ be the maximum width of a row containing an element of $A$. Let

$$
\mathfrak{S}_{A}=\left\{\sigma \in \mathfrak{S}_{d}: \sigma \text { preserves } A \text { and fixes } T_{0} \backslash A\right\} .
$$


When $|A|>w_{A}$, define a Garnir operator as an element of $\mathbb{C S}_{d}$ by

$$
G_{A}=\sum_{\sigma \in \mathfrak{S}_{A}} \sigma
$$

For an example of a Garnir operator acting on a tableau, see Section 3.5.

Let $\mathcal{F}_{\lambda, n}$ be the formal $\mathbb{C}$-span of symbols $T \in \mathcal{T}_{\lambda, n}$ and let $\mathcal{R}_{\lambda, n}$ be the subspace of $\mathcal{F}_{\lambda, n}$ generated by all

$$
T_{1}-T_{2} \text {, where } T_{1} \text { and } T_{2} \text { agree up to a row permutation, }
$$

and

$$
G_{A}(T), \text { where } A \subset T_{0} \text { with }|A|>w_{A} .
$$

Theorem 1. As $G L(V)$-modules, we have

$$
\mathcal{F}_{\lambda, n} / \mathcal{R}_{\lambda, n} \cong \mathbb{S}_{\lambda}(V)
$$

Proof. The map is induced by $T \mapsto e_{T}$. See, for example, $[5, \S 8]$, where the convention is transpose to ours.

\section{Constructing the Pieri Inclusion for Removing One Box}

\subsection{Notation and Set-Up}

We will write $\lambda=\left(\lambda_{1}, \ldots, \lambda_{r}\right)$ in block form as $\lambda=\left(w_{1}^{h_{1}}, \ldots, w_{N}^{h_{N}}\right)$, where $w_{i}<w_{i+1}$ and exactly $h_{i}$ parts of $\lambda$ are equal to $w_{i}$. That is, $N$ is the number of blocks in $\lambda$, where block 1 is the lowest geometrically, $w_{b}$ is the width of block $b$, and $h_{b}$ is the height of block $b$. See Figure 1. For example, we will write $(5,2,2,2,1,1)$ as $\left(1^{2}, 2^{3}, 5\right)$,

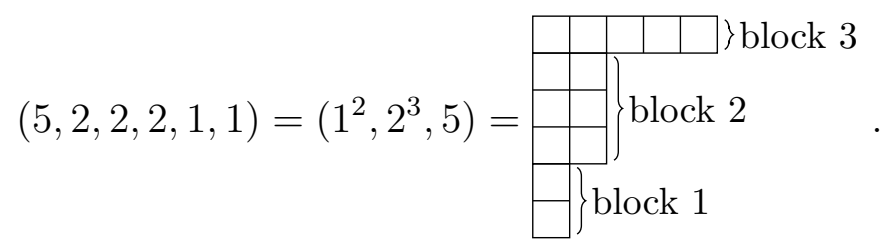

For any box $x \in T_{0}$ at the bottom right of some block $k$, i.e. so that $\lambda \backslash\{x\}$ is still a diagram, we will define the map

$$
\Phi_{1}: \mathcal{F}_{\lambda, n} \rightarrow V \otimes \mathcal{F}_{\lambda \backslash\{x\}, n}
$$

on a basis and then show that $\Phi_{1}$ descends to a $G L(V)$-module map.

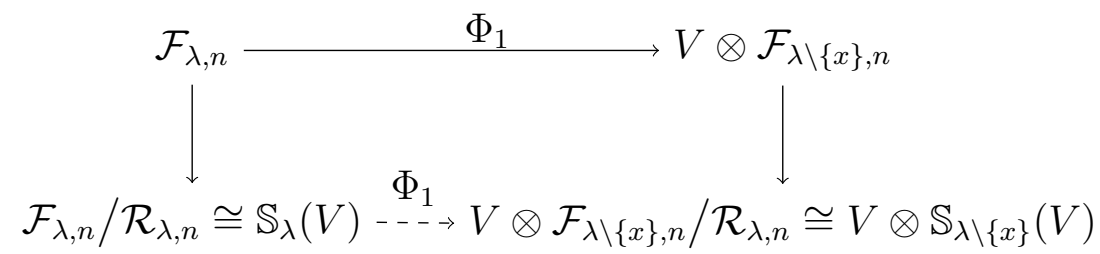




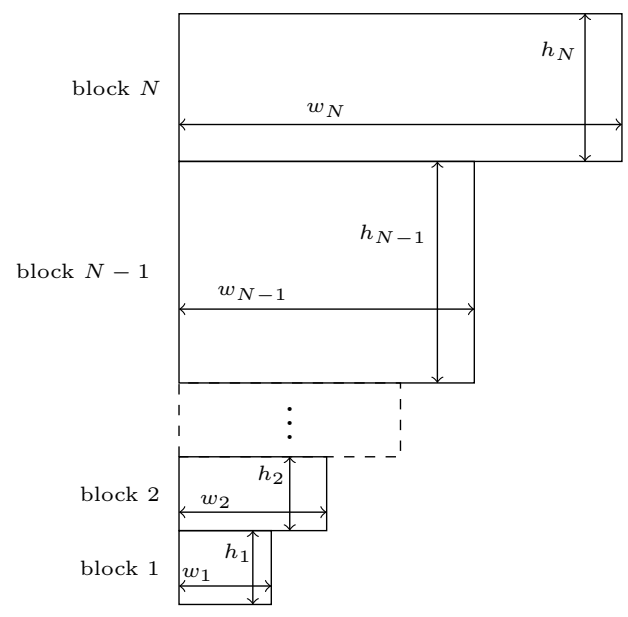

Figure 1: The shape $\lambda$ with $N$ blocks.

We first introduce further notation. For a given shape $\lambda$ let $[b]$ denote the $b$ th block, $[b](i)$ denote the $i$ th row of the bth block, and $[b](i, j)$ denote the box in block $b$, row $i$, and column $j$. We will count blocks and rows from the bottom and columns from the left, so that [1] is the bottom-most block in the shape, [b](1) is the bottom-most row of $[b]$, and $[b](r, 1)$ is the left-most box in $[b](r)$. We write

$$
[b](i, j) \leqslant[c](k, l)
$$

if $[b](i, j)$ is geometrically (weakly) lower than $[c](k, l)$, i.e. $b<c$ or $b=c$ and $i \leqslant k$. The strict inequality is defined similarly. We will extend this notation to compare rows and blocks in the natural way. For a given $T \in \mathcal{T}_{\lambda, n}$, we denote the entry in box $[b](i, j)$ by $T_{[b](i, j)}$.

Example 2. If $\lambda=\left(1^{2}, 3^{4}, 4^{1}, 6^{2}\right)$ and

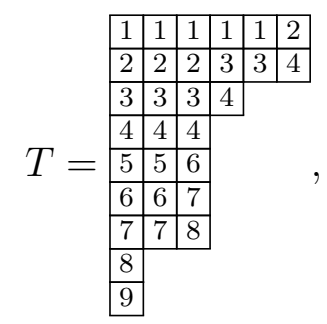

then $T_{[1](1,1)}=9, T_{[2](3,2)}=5$, and $T_{[4](2,6)}=2$.

\subsection{Evacuation Routes}

An evacuation route $R$ is a selection of a string of boxes starting from the bottom of some block. The selection does not need to contain a box from every row, contiguous rows at the top of blocks and even entire blocks can be skipped. However, an evacuation route cannot "skip rows within a block." Formally, we have the following definition. 
Definition 3 (Evacuation Route). An evacuation route $R$ starting at $\left[b_{0}\right]$ is a subset of boxes in $T_{0}$ such that $R$ contains a box in row $\left[b_{0}\right](1), R$ contains at most one box per row, and if $[b](i, j) \in R$, then $[b]\left(k, j_{k}\right) \in R$ for all $1 \leqslant k<i$ and some $1 \leqslant j_{k} \leqslant w_{b}$.

Example 4. An evacuation route starting at [1] on $\left(1^{2}, 3^{4}, 4^{1}, 6^{2}\right)$ is given by the shaded boxes in the diagram below. Note that while there is no box selected from row [2](3) this row is not skipped since there is no box selected above this row within the third block.

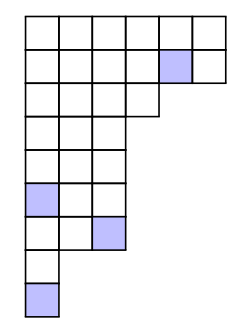

Non-Example 5. The shaded selection of boxes below is not an evacuation route on $\left(1^{2}, 3^{4}, 4^{1}, 6^{2}\right)$ since row $[2](2)$ has been skipped, indicated by " $\times$ ". That is, no box is selected from row $[2](2)$ but a box is selected from row [2](3).

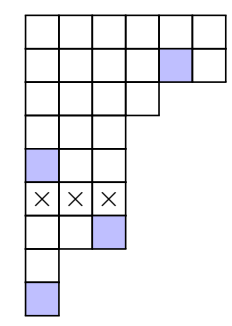

\subsection{1-Paths}

A 1-path $P$ on $\lambda$ moves boxes up the diagram via some associated evacuation route $R^{P}$. We will treat a 1-path as acting on general shapes, where the highest box in $R^{P}$ is "removed" by the 1-path and viewed as being moved to the box $[N+1](1,1)$ attached to the top of $T_{0}$. Below we illustrate a 1-path moving boxes up via an evacuation route. We highlight only the boxes in the evacuation route.

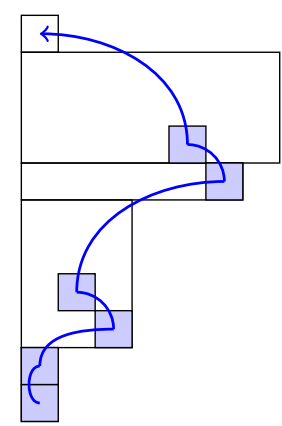

Formally, we have the following definition. 
Definition 6 (1-Path). Let $X=\left\{x_{1}:=\left[b_{1}\right]\left(1, w_{b_{1}}\right)\right\}$ and $Y=\left\{y_{1}:=[N+1](1,1)\right\}$, where $Y$ is viewed as block $[N+1]$ attached to the top of $T_{0}$. A 1-path $P$ removing $X$ is a map of boxes $P: \lambda \cup Y \rightarrow \lambda \cup Y$ along with an evacuation route $R=R^{P}$ such that the following hold.

- $R$ starts at $\left[b_{1}\right]$. Note that $R$ can contain $x_{1}$, though this is not a requirement.

- $P$ is geometrically increasing on rows, i.e., for all boxes $x \in \lambda \cup Y, x \leqslant P(x)$, with $P$ strictly geometrically increasing on $R$.

- If $R_{1}$ is the orbit of $x_{1}$ under $P^{\mathbb{N}}$, then $y_{1} \in R_{1}$ and $R \cup X \cup Y=R_{1}$.

- $P$ preserves row order in $R$ within blocks. That is, if $[b](i, j),[b](k, l) \in R$ with $i<k$ and $P([b](i, j)), P([b](k, l)) \in[b]$, then $P([b](i, j))<P([b](k, l))$.

- $P$ fixes those boxes not in $R$ or $X$, i.e. $P=\operatorname{id}_{\lambda \cup Y}$ except on $R \cup X$, and $P(R)=$ $R \backslash X \cup Y$.

Example 7. Consider the evacuation route $R$ starting at [1] on $\lambda=\left(1^{2}, 3^{4}, 4^{1}, 6^{2}\right)$ given in Example 4. Let $X=\{[1](1,1)\}, Y=\{[5](1,1)\}$, and define the 1-path $P: \lambda \cup Y \rightarrow \lambda \cup Y$ removing $X$ to be the map on boxes given by

$$
\begin{array}{ll}
P(([1](1,1))=[2](1,3), & P(([2](1,3))=[2](2,1), \\
P(([2](2,1))=[4](1,5), & P(([4](1,5))=[5](1,1),
\end{array}
$$

and $P=\operatorname{id}_{\lambda \cup Y}$ otherwise. We can identify $P$ and the evacuation route $R=R^{P}$ pictorially by the diagram below.

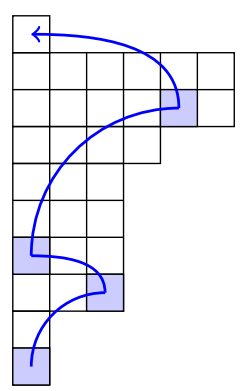

\subsection{Defining the Pieri Inclusion Removing One Box}

We now define the components of the formulation of the Pieri inclusion $\Phi_{1}$ removing one box. For a 1-path $P$ removing $X$ with evacuation route $R^{P}$, let $h^{P}$ be the number of rows in $R^{P}$ and $(-1)^{P}:=(-1)^{h^{P}}$. For $b=b_{1}, \ldots, N$, let $h_{b}^{P}$ be the number of rows in $R^{P} \cap[b]$. Recall that $w_{b}$ is the width of block $[b]$ and, for $b \geqslant b_{1}+1$, define $h(b)=w_{b}-w_{b-1}+h_{b-1}^{P}$ to be the hook length of block $b$, and for $b=b_{1}+1, \ldots, N$ define

$$
H(b)=\sum_{j=b_{1}+1}^{b} h(j) .
$$


For $b=b_{1}+1, \ldots, N$, let

$$
H_{b}(P)=\left\{\begin{array}{ll}
1 & \text { if } R^{P} \cap[b]=\emptyset \\
H(b) & \text { otherwise }
\end{array},\right.
$$

and let $H_{b_{1}}(P)=1$. Then define

$$
H(P)=\prod_{b=b_{1}}^{n} H_{b}(P) .
$$

Example 8. Continuing Example 7, let $\lambda=\left(1^{2}, 3^{4}, 4^{1}, 6^{2}\right), X=\{[1](1,1)\}$, and $P$ as in the diagram below.

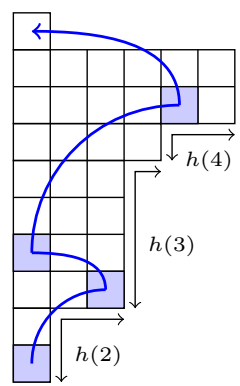

Then for the shape $\lambda$ we have

$$
\begin{gathered}
h(2)=4, \quad h(3)=5, \quad h(4)=3, \\
H(1)=1, \quad H(2)=h(2)=4, \quad H(3)=h(3)+h(2)=9, .
\end{gathered}
$$

and

$$
H(4)=h(4)+h(3)+h(2)=12 .
$$

For this 1-path $P$ we have

$$
H_{1}(P)=1, \quad H_{2}(P)=4, \quad H_{3}(P)=1, \text { and } \quad H_{4}(P)=12,
$$

and so

$$
H(P)=\prod_{i=1}^{4} H_{i}(P)=48 .
$$

Also note that the evacuation route $R^{P}$ contains boxes in four different rows in the diagram, for this 1-path we have $(-1)^{p}=(-1)^{4}=1$.

For $T \in \mathcal{T}_{\lambda, n}$, denote by $\alpha_{1}^{P}$ the entry in the box $P^{-1}\left(y_{1}\right) \in T$ and extend $P$ to $T$ by acting on the entries, with the image

$$
P(T)=e_{\alpha_{1}^{P}} \otimes T_{P} \in V \otimes \mathcal{T}_{\lambda \backslash X, n},
$$

where $T_{P} \in \mathcal{T}_{\lambda \backslash X, n}$ is defined by $\left(T_{P}\right)_{[b](i, j)}=T_{P^{-1}([b](i, j))}$. To ease notation we will write

$$
\alpha_{1}^{P} \quad \text { in place of } \quad e_{\alpha_{1}^{P}}
$$

in the image $P(T)$. 
Definition $9\left(\boldsymbol{\Phi}_{1}(\boldsymbol{T})\right)$. For $T \in \mathcal{T}_{\lambda, n}$, define $\Phi_{1}(T)$ by

$$
\Phi_{1}(T)=\sum_{P} \frac{(-1)^{P}}{H(P)} P(T),
$$

where the sum is over all 1-paths $P$ removing $X$, and define the map $\Phi_{1}: \mathcal{F}_{\lambda, n} \rightarrow$ $V \otimes \mathcal{F}_{\lambda \backslash X, n}$ by extending linearly.

Example 10. Let $T$ be as in Example 2 and $P$ be as in Example 8. The single term in the image $\Phi_{1}(T)$ corresponding to this 1-path can be visualized via the diagram below.

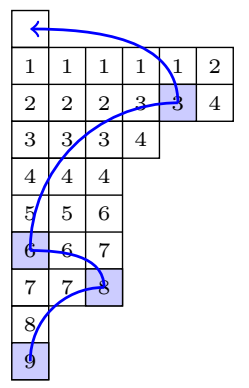

This gives

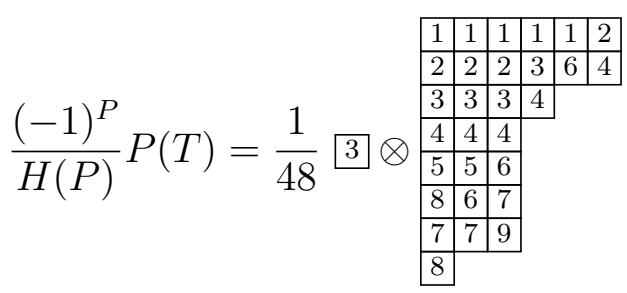

where $3=e_{3} \in V$.

\subsection{Showing the Straightening in an Example of One Box Removal}

We now compute the straightening from Section 1.4. If

$$
A_{1}=\{[2](1,1),[2](1,2),[1](2,1)\}=\square
$$

then

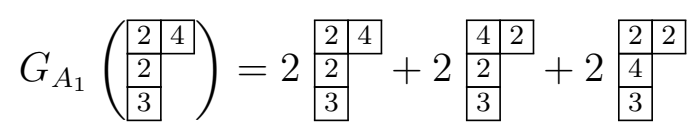

and, modulo $\mathcal{R}_{(2,1,1), 4}$

$$
\begin{array}{|l|l|l|}
\hline 2 & 4 \\
\hline 2 & =-\frac{1}{2} & \begin{array}{|l|l|}
\hline 2 & 2 \\
\hline 4 & \\
\hline 3
\end{array}
\end{array} .
$$

If

$$
A_{2}=\{[1](1,1),[1](2,1)\}=\square,
$$


then

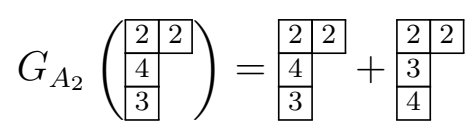

and, modulo $\mathcal{R}_{(2,1,1), 4}$,

$$
\begin{array}{|l|l|l|}
\hline 2 & 2 \\
\hline 4 & 2 & 2 \\
\hline 3 & -3 & \\
\hline 4 &
\end{array}
$$

So, modulo $\mathcal{R}_{(2,1,1), 4}$,

$$
\begin{array}{|l|l|l|l|}
\hline 2 & 4 \\
\hline 2 & =\frac{1}{2} & 2 & 2 \\
\hline 3 & 3 \\
\hline 4
\end{array}
$$

and thus as elements of $\mathcal{F}_{(2,1,1), 4} / \mathcal{R}_{(2,1,1), 4}$ we have

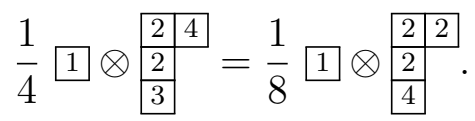

Similarly, as elements of $\mathcal{F}_{(2,1,1), 4} / \mathcal{R}_{(2,1,1), 4}$, we have

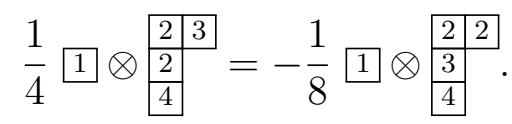

Note that in this example the terms that were straightened cancelled with each other and so did not appear in the image. This is not the case in general.

\section{Constructing the Pieri Inclusion for Removing Many Boxes}

\subsection{Notation and Set-Up}

Let $X=\left\{x_{1}=\left[b_{1}\right]\left(1, w_{b_{1}}\right), \ldots, x_{m}=\left[b_{m}\right]\left(i_{m}, w_{b_{m}}\right)\right\}$ be a set of $m$ boxes in $\lambda$ with $x_{i}<x_{i+1}$ so that removing the boxes in $X$ from $T_{0}$ gives a Young diagram and let $\lambda \backslash X$ be the associated partition. We call such a set $X$ a removal set for $T_{0}$ (or for $\lambda$ ). As before, we will define the map $\Phi_{m}: \mathcal{F}_{\lambda, n} \rightarrow \bigwedge^{m} V \otimes \mathcal{F}_{\lambda \backslash X, n}$ on a basis and then show that $\Phi_{m}$ is a $G L(V)$-map.

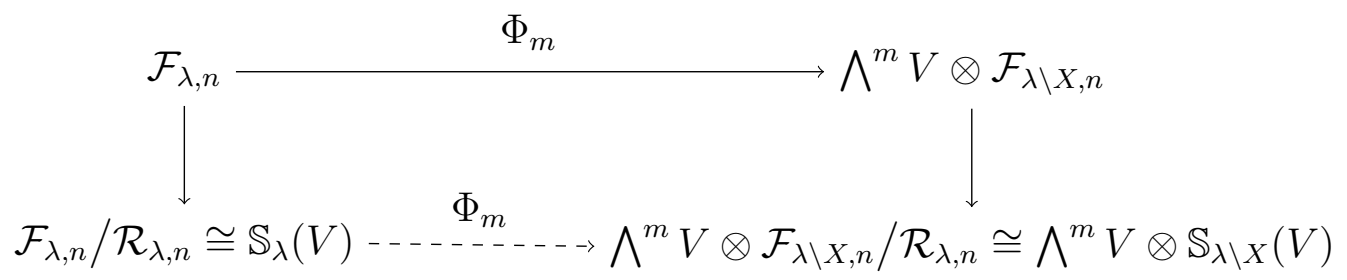

\section{$4.2 m$-Paths}

Extending the notion of a 1-path, an $m$-path on $\lambda$ is a map of boxes that moves boxes up the diagram via some associated evacuation route with $m$ interlaced orbits. We will formally define this interlacing property in Definition 11, but will first illustrate it with 
some examples. As with 1-paths, we treat $m$-paths as acting on general shapes, where the highest $m$ boxes in $R^{P}$ are "removed" by the $m$-path and viewed as being moved to the boxes $[N+1](1,1), \ldots,[N+1](m, 1)$ attached to the top of $T_{0}$. An example of a 2-path is pictured below. We highlight only the boxes in the evacuation route.

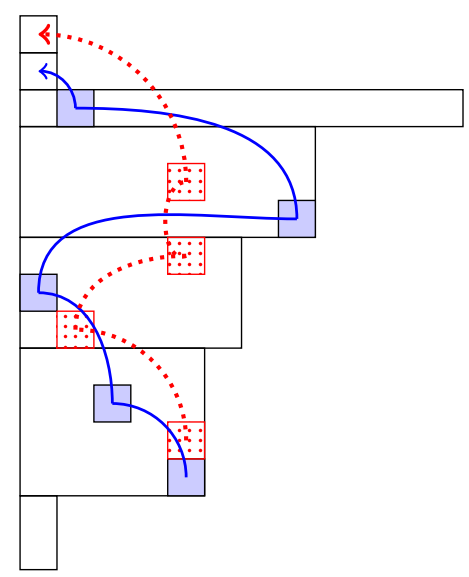

The interlacing property for $m$-paths is not so strict as the above example suggests. We require that an $m$-path interlaces orbits only within blocks while multiple orbits are present. This is illustrated further in the example below.

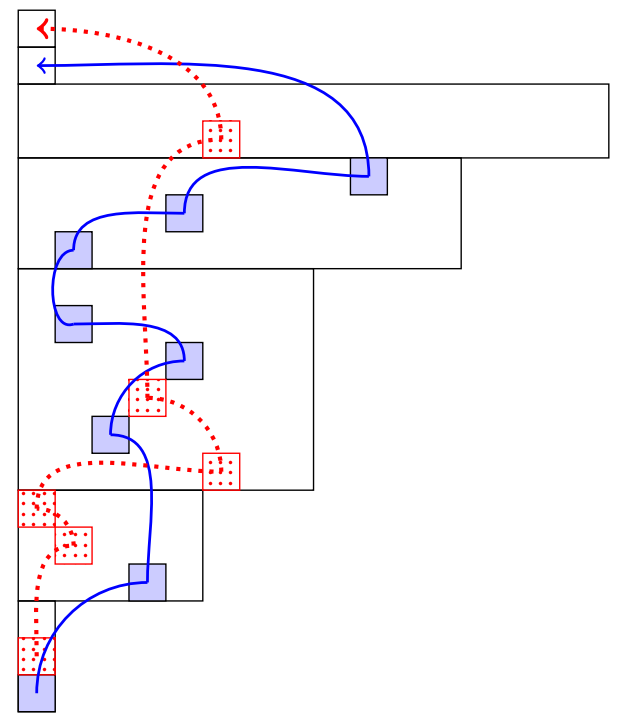

Formally, we have the following definition.

Definition 11 ( $\boldsymbol{m}$-Path). Let $X=\left\{x_{1}=\left[b_{1}\right]\left(1, w_{b_{1}}\right), \ldots, x_{m}=\left[b_{m}\right]\left(i_{m}, w_{b_{m}}\right)\right\}$ be a removal set for $T_{0}$ and $Y=\left\{y_{1}:=[N+1](1,1), \ldots, y_{m}:=[N+1](m, 1)\right\}$, where $Y$ is viewed as block $N+1$ attached to the top of $T_{0}$. An $m$-path $P$ removing $X$ is a map of boxes $P: \lambda \cup Y \rightarrow \lambda \cup Y$ along with an evacuation route $R=R^{P}$ such that the following hold.

- $R$ starts at $\left[b_{1}\right]$. Note that $R$ can intersect $X$, though this is not a requirement. 
- $P$ is geometrically increasing on rows, i.e., for all boxes $x \in \lambda \cup Y, x \leqslant P(x)$, with $P$ strictly geometrically increasing on $R$.

- If $R_{i}$ is the orbit of $x_{i}$ under $P^{\mathbb{N}}$, then $y_{i} \in R_{i}$ and $R \cup X \cup Y=\bigsqcup_{i=1}^{m} R_{i}$.

- If there are $k$ distinct orbits in a block, then the first $k$ rows of that block must be in different orbits. i.e., if $R_{i_{1}}^{P}, \ldots, R_{i_{k}}^{P}$ intersect some block [b], then for $j=1, \ldots k$, up to relabeling, $R_{i_{j}}^{P} \cap[b](j) \neq \emptyset$.

- $P$ preserves row order in $R$ within blocks, and so interlaces orbits. That is, if $[b](i, j),[b](k, l) \in R$ with $i<k$ and $P([b](i, j)), P([b](k, l)) \in[b]$, then $P([b](i, j))<$ $P([b](k, l))$.

- $P$ fixes those boxes not in $R$ or $X$, i.e. $P=\operatorname{id}_{\lambda \cup Y}$ except on $R \cup X$, and $P(R)=$ $R \backslash X \cup Y$.

\subsection{Defining the Pieri Inclusion Removing Many Boxes}

For an $m$-path $P$ with evacuation route $R^{P}$, let $h^{P},(-1)^{P}, h_{b}^{P}$, and $H(b)$ be defined as in Section 3.4. For $b=b_{1}+1, \ldots, N$, let $H_{b}(P)=1$ if $R^{P} \cap[b]=\emptyset$ and let $H_{b_{1}}(P)=1$. If $b \geqslant b_{1}+1$ and $\left|R^{P} \cap[b]\right|=k_{b} \neq 0$, then let

$$
H_{b}(P)=\prod_{i=1}^{k_{b}}(H(b)-(m-i))
$$

and define

$$
H(P)=\prod_{b=b_{1}}^{n} H_{b}(P) .
$$

For $T \in \mathcal{T}_{\lambda, n}$, denote by $\alpha_{i}^{P}$ the entry in the box $P^{-1}\left(y_{i}\right) \in T$ and extend $P$ to $T$ by acting on the entries, with the image

$$
P(T)=e_{\alpha_{1}^{P}} \wedge \cdots \wedge e_{\alpha_{m}^{P}} \otimes T_{P} \in \bigwedge^{m} V \otimes \mathcal{T}_{\lambda \backslash X, n},
$$

where $T_{P} \in \mathcal{T}_{\lambda \backslash X, n}$ is defined by $\left(T_{P}\right)_{[b](i, j)}=T_{P^{-1}([b](i, j))}$. To ease notation we will write

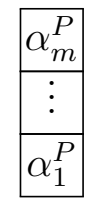

in place of $\quad e_{\alpha_{1}^{P}} \wedge \cdots \wedge e_{\alpha_{m}^{P}}$

in the image $P(T)$.

Definition $12\left(\boldsymbol{\Phi}_{\boldsymbol{m}}(\boldsymbol{T})\right)$. For $T \in \mathcal{T}_{\lambda, n}$, define $\Phi_{m}(T)$ by

$$
\Phi_{m}(T)=\sum_{P} \frac{(-1)^{P}}{H(P)} P(T),
$$

where the sum is over all $m$-paths $P$ removing $X$, and define the map $\Phi_{m}: \mathcal{F}_{\lambda, n} \rightarrow$ $\bigwedge^{m} V \otimes \mathcal{F}_{\lambda \backslash X, n}$ by extending linearly. 


\subsection{An Example of Two Box Removal}

We now compute an example of the Pieri inclusion when $m=2$ and $n=6$. The Schur-Weyl module $\mathbb{S}_{\left(1^{4}, 2^{2}\right)}(V)$ appears as a summand in the decomposition of $\mathbb{S}_{\left(1^{2}\right)}(V) \otimes$ $\mathbb{S}_{\left(1^{2}, 2^{2}\right)}(V)$,
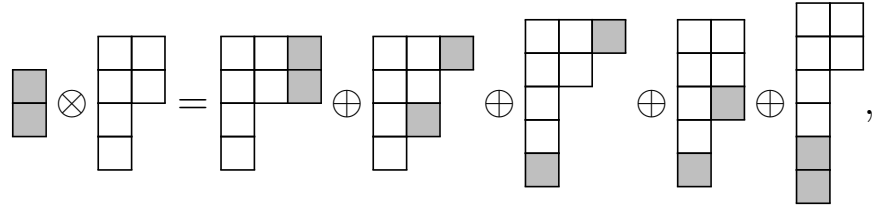

and so we can consider the Pieri inclusion

$$
\mathbb{S}_{\left(1^{4}, 2^{2}\right)}(V) \stackrel{\Phi_{2}}{\longrightarrow} \mathbb{S}_{\left(1^{2}\right)}(V) \otimes \mathbb{S}_{\left(1^{2}, 2^{2}\right)}(V)
$$

We will compute the image of the highest weight vector under this map,

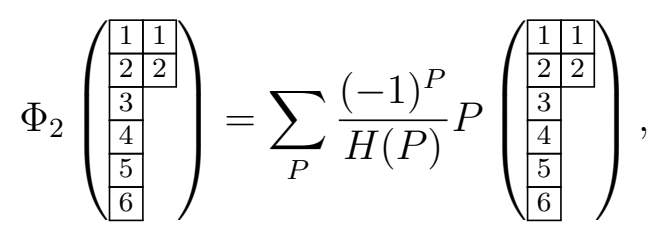

where the sum is over all 2-paths $P$ on $\left(1^{4}, 2^{2}\right)$ removing $X=\left\{x_{1}=[1](1,1), x_{2}=\right.$ $[1](2,1)\}$. To compute the image we divide the 2 -paths in the sum in to three groups according to their values for $H(P)$. For each term we illustrate the 2-path with arrows and shade the boxes in the corresponding evacuation route while distinguishing the orbits of $x_{1}$ and $x_{2}$. As in Sections 1.3 and 1.4, we give the images up to row permutations and we star the paths whose images require straightening.

As $H(P)$ depends on the number of orbits in each block, we first consider all 2paths with corresponding evacuation routes contained in block [1]. All such 2-paths have $H(P)=1$. Recall that the sign $(-1)^{P}$ depends on the number of rows in the corresponding evacuation route.
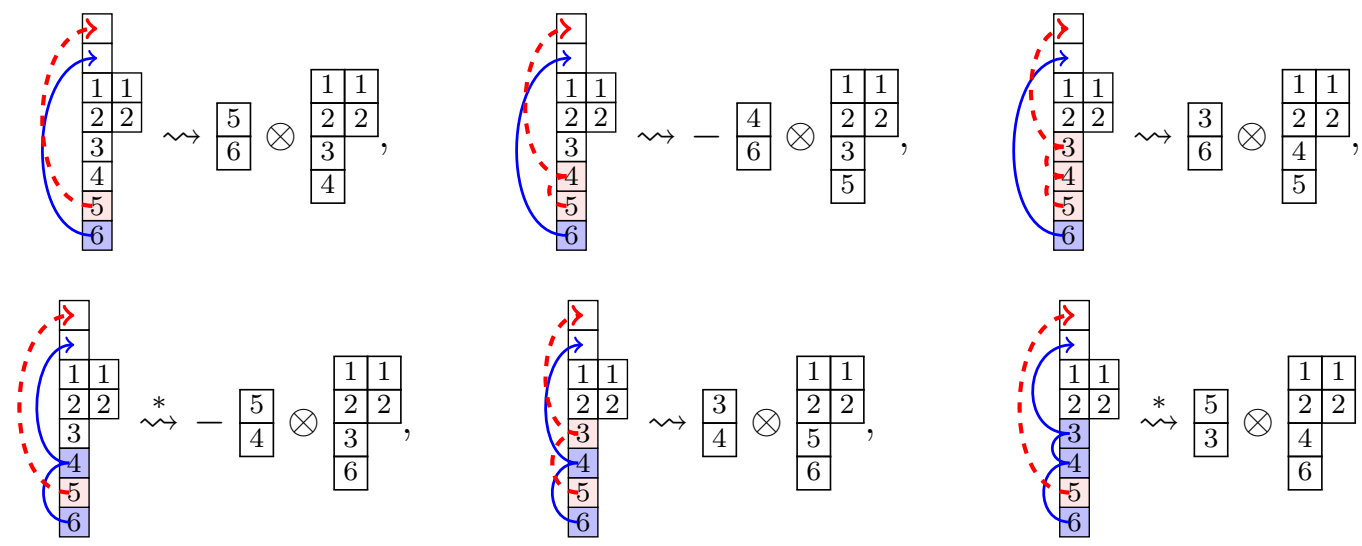

We next consider the 2-paths where exactly one orbit intersects block [2], which all 
have $H(P)=H_{2}(P)-1=5-1=4$. Again, recall that the sign $(-1)^{P}$ depends on the number of rows in the corresponding evacuation route. For paths hitting rows [2](1) or $[2](2)$, we only show the 2-paths that hit the first column of these rows, as the paths that hit the second column in either row will give the same result up to row permutation. We will include the multiple (two or four) in the coefficients of the terms below.

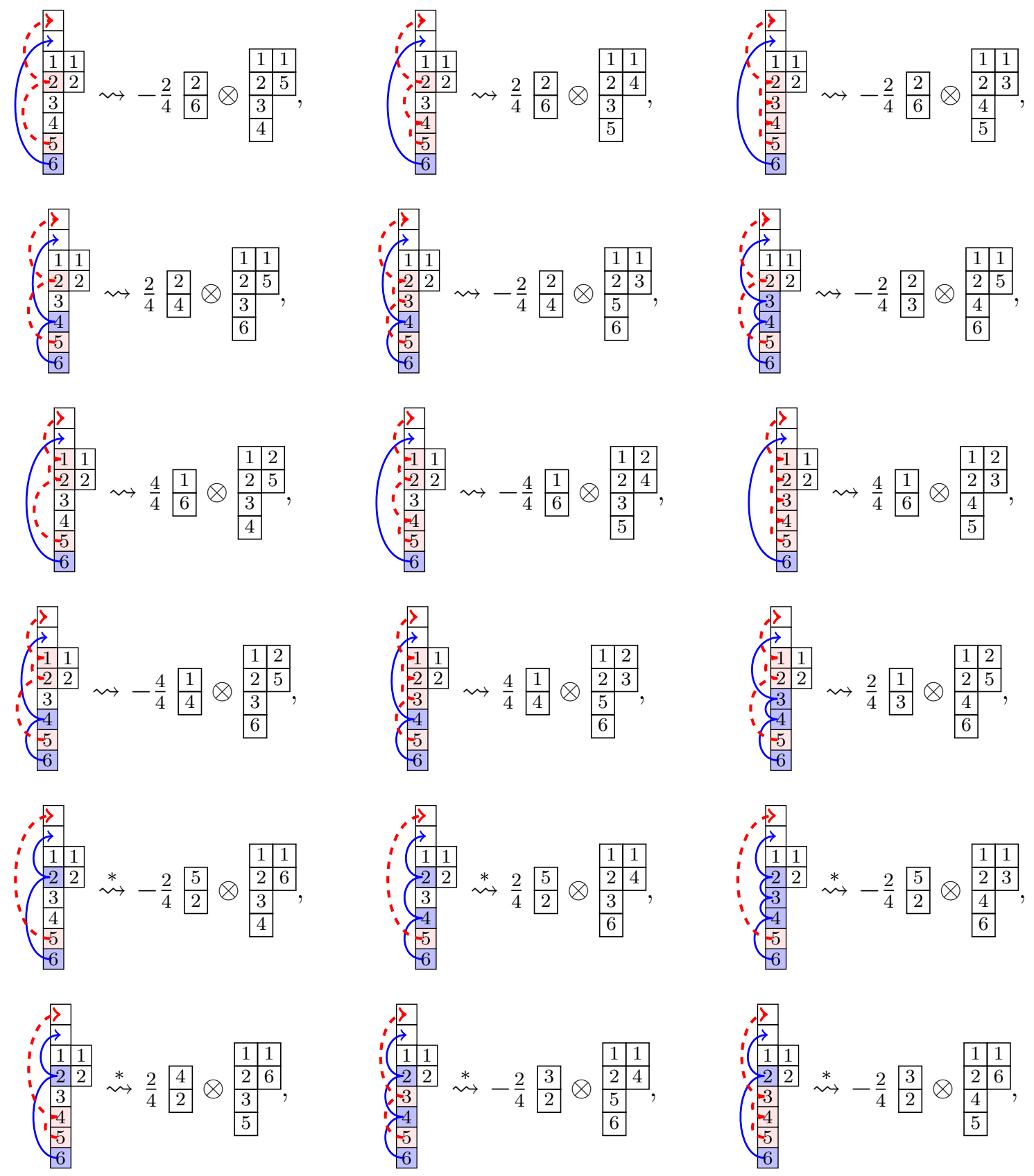




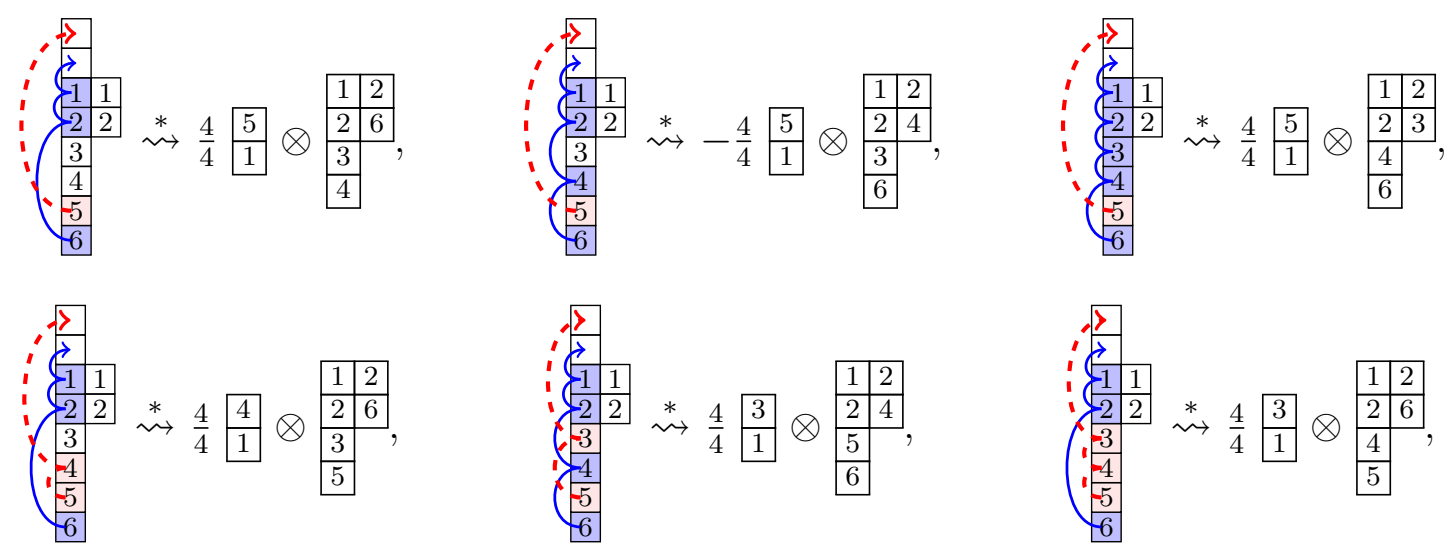

For all remaining 2-paths the orbits of both $x_{1}$ and $x_{2}$ intersect block [2], and so $H(P)=H_{2}(P) \cdot\left(H_{2}(P)-1\right)=5 \cdot(5-1)=5 \cdot 4$. Again, recall that the sign $(-1)^{P}$ depends on the number of rows in the corresponding evacuation route and that we only show the 2-paths that hit the first column in block [2].

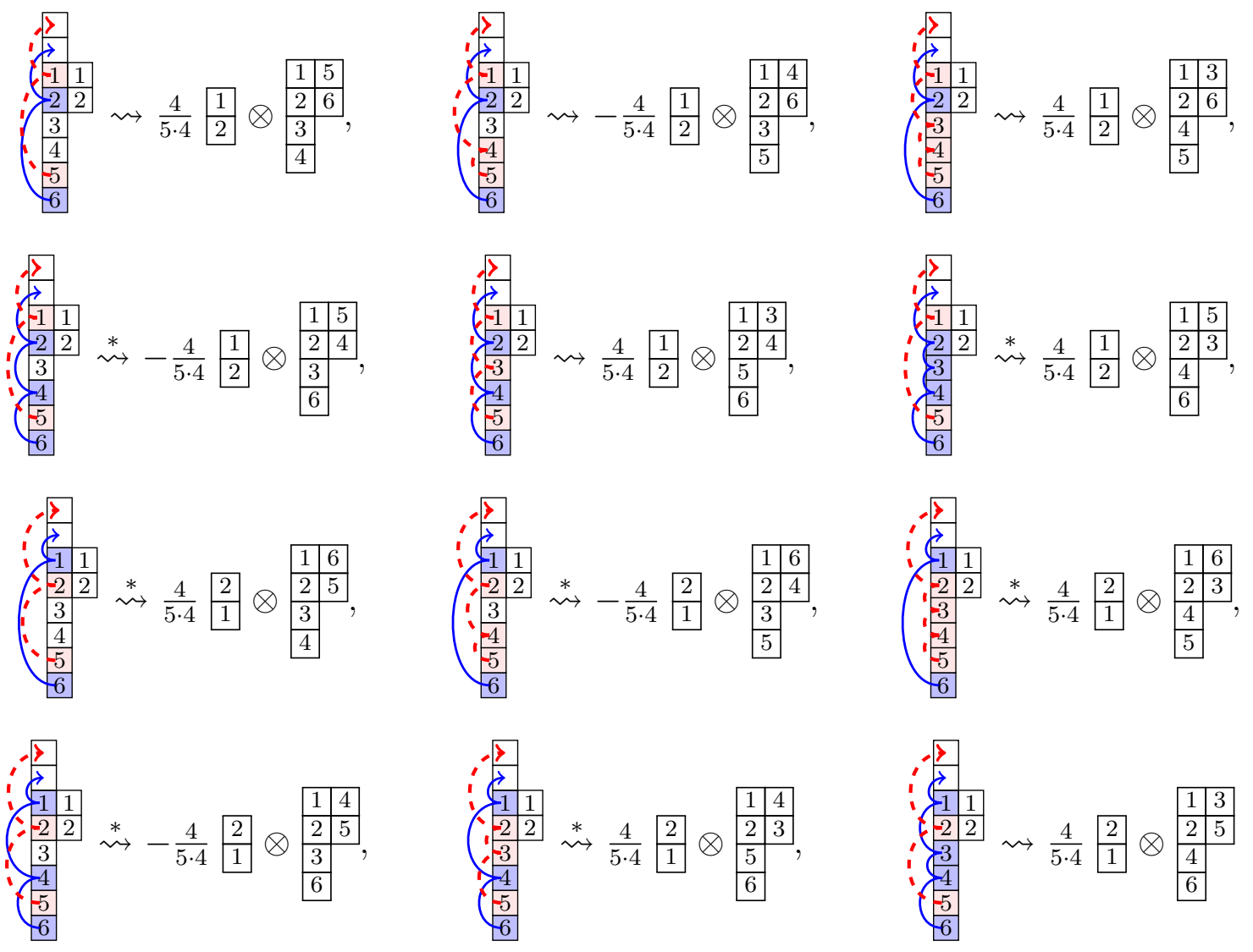


We now compute the straightening for the term

$$
-\frac{4}{5 \cdot 4} \begin{array}{l|l|l|l|}
\hline 1 \\
\hline 2
\end{array} \otimes \begin{array}{|l|l|}
\hline 2 & 5 \\
\hline 3 & 4 \\
\hline 6
\end{array}
$$

If

$$
A_{1}=\{[2](1,1),[2](1,2),[2](2,2)\}=\square
$$

then

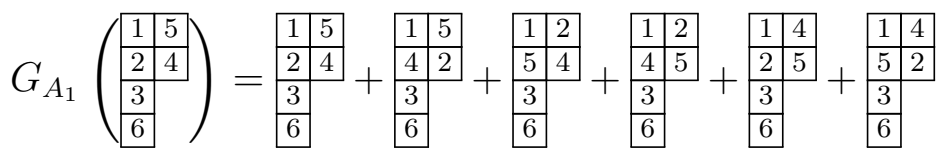

and, modulo $\mathcal{R}_{(2,2,1,1), 6}$,

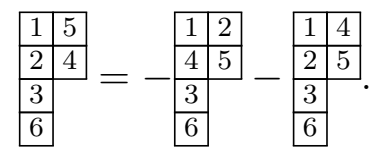

If

$$
A_{2}=\{[2](1,1),[2](1,2),[1](2,1)\}=\square,
$$

then

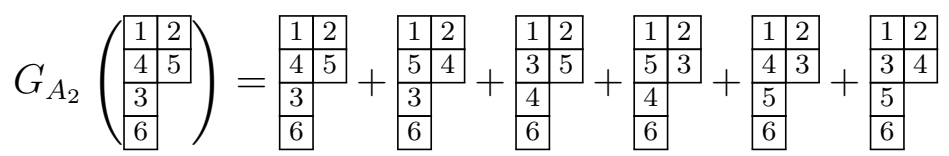

and, modulo $\mathcal{R}_{(2,2,1,1), 6}$,

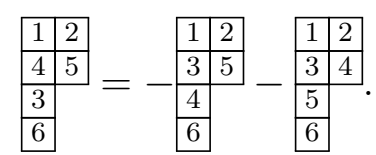

Thus, modulo $\mathcal{R}_{(2,2,1,1), 6}$,

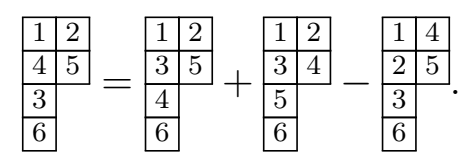

Similarly, via straightening we have, modulo $\mathcal{R}_{(2,2,1,1), 6}$,

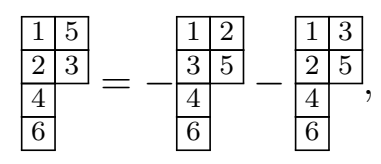

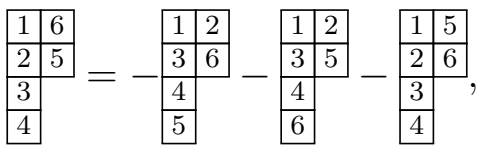

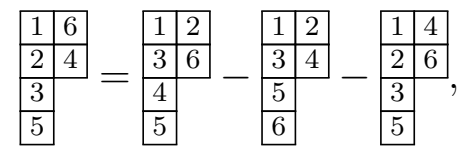




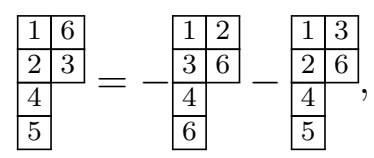

and

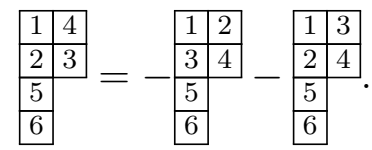

Recall that in the image of each two path $\frac{\alpha}{\beta}=e_{\alpha} \wedge e_{\beta} \in \bigwedge^{2} V$, and so

$$
\frac{\alpha}{\beta}=-\frac{\beta}{\alpha} .
$$

Thus,

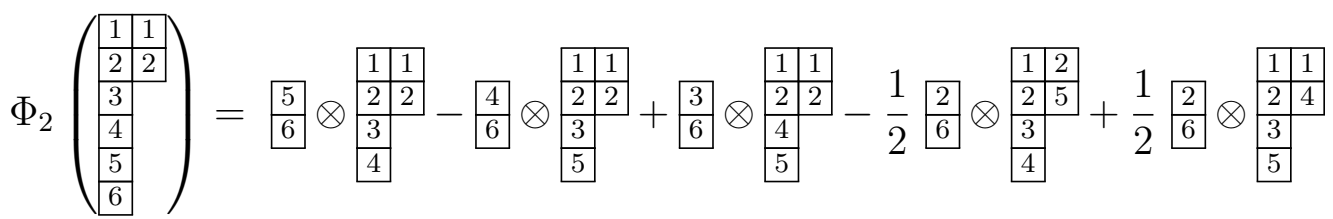

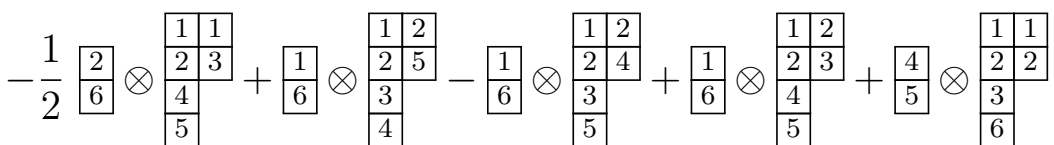

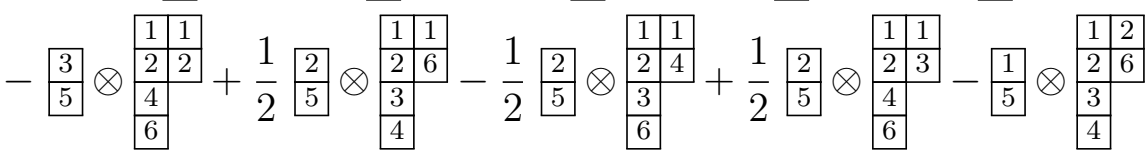

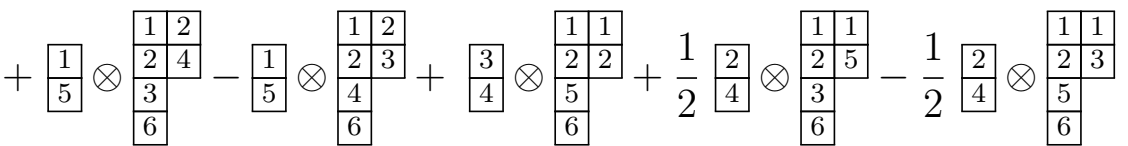

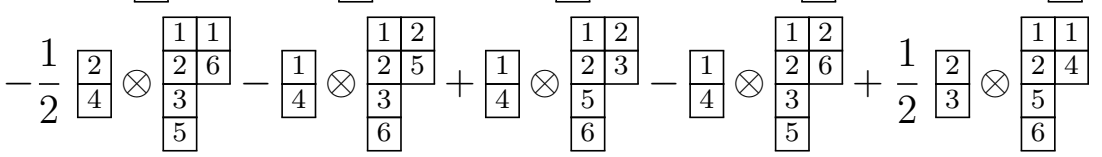

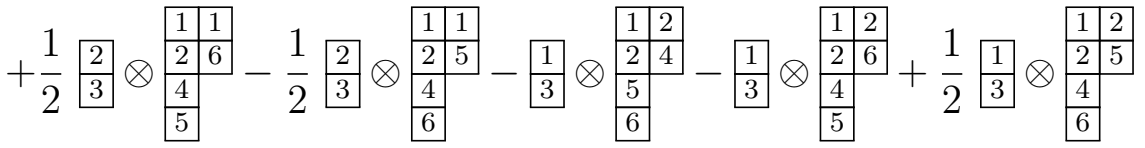

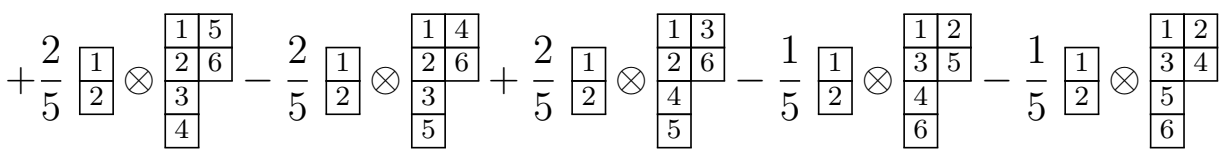

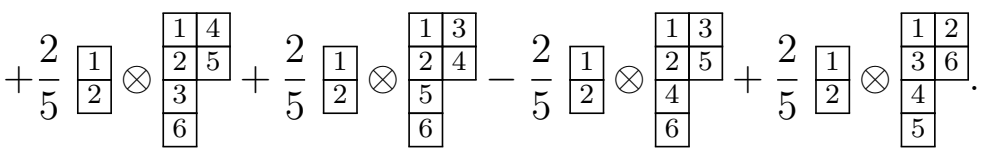

\section{Generating Garnir Operators and Tools for Collapsing Sums}

\subsection{Hooks and Generating Garnir Operators}

To show that $\Phi_{m}$ is a $G L(V)$-map, we will need to show that $\Phi_{m}\left(\mathcal{R}_{\lambda, n}\right) \subset \bigwedge^{m} V \otimes \mathcal{R}_{\lambda \backslash X, n}$. It will be straightforward to show that $\Phi_{m}$ preserves equivalence up to a row permutation 
(Property 1), but it will take some work to show that $\Phi_{m}$ preserves Garnir operators (Property 2). In this section we make this future proof (in Sections 6 and 7 ) easier by showing that all Garnir operators can be generated up to row permutation by Garnir operators over certain sets.

We will show that all Garnir operators are generated up to row permutation by Garnir operators of minimal size, i.e. those $G_{A}$ with $|A|=w_{A}+1$ (where $w_{A}$ is as in Section 2). We then show that all Garnir operators of minimal size are themselves generated up to row permutation by Garnir operators over hooks, which are those $G_{A}$ where $A$ is of minimal size and consists of exactly a complete row and one other box. We start by formalizing the idea of a hook.

Definition 13 (Hook). We say that $A \subset T_{0}$ is a hook if for some row $[b](r)$,

$$
A=[b](r) \cup\left\{a_{0}\right\}
$$

where

$$
a_{0}=\left\{\begin{array}{ll}
{[b](r-1,1)} & \text { if } r \neq 1 \\
{[b-1]\left(h_{b-1}, 1\right)} & \text { if } r=1
\end{array} .\right.
$$

That is,

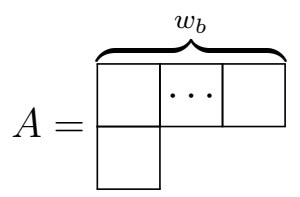

Theorem 14. Let $T \in \mathcal{T}_{\lambda, n}$ and $A \subset T_{0}$ such that $|A|>w_{A}$. Then $G_{A}(T)$ is generated up to row permutation by $\left\{G_{A^{\prime}}\left(T^{\prime}\right) \mid T^{\prime} \in \mathcal{F}_{\lambda, n}\right.$ and $A^{\prime}$ is a hook $\}$.

\subsection{Generating Garnir Operators Using Minimal Size Sets}

To prove Theorem 14, we first show that $G_{A}(T)$ is generated by Garnirs of minimal size for any $T \in \mathcal{T}_{\lambda, n}$ and any $A \subset T_{0}$ with $|A|>w_{A}$. We will also show that if $|A|>w_{A}+1$, then $G_{A}(T)$ is generated by Garnirs over $A \backslash\{y\}$ for any $y \in A$.

Lemma 15. Let $T \in \mathcal{T}_{\lambda, n}$. If $A \subset T_{0}$ with $|A|>w_{A}$, then for any $x \in T_{0}$ such that $|A \cup\{x\}|>w_{A \cup\{x\}}$,

$$
G_{A \cup\{x\}}(T) \in\left\langle G_{A}\left(T^{\prime}\right): T^{\prime} \in \mathcal{T}_{\lambda, n}\right\rangle .
$$

Proof. Let $A \subset T_{0}$ with $|A|>w_{A}$ and $x \in T_{0} \backslash A$. For all $y \in A \cup\{x\}$, let $\tau_{x, y}$ be the permutation that switches $x$ and $y$ and fixes the rest of $A \cup\{x\}$. Then for any $\sigma \in \mathfrak{S}_{A \cup\{x\}}$,

$$
\sigma(y)=x \Longleftrightarrow\left(\sigma \tau_{x, y}\right)(x)=x \Longleftrightarrow \sigma \tau_{x, y} \in \mathfrak{S}_{A} .
$$

Then,

$$
G_{A \cup\{x\}}(T)=\sum_{\sigma \in \mathfrak{S}_{A \cup\{x\}}} \sigma T
$$




$$
\begin{aligned}
& =\sum_{y \in A \cup\{x\}} \sum_{\substack{\sigma \in \mathfrak{S}_{A \cup\{x\}} \\
\text { s.t. } \sigma(y)=x}} \sigma T \\
& =\sum_{y \in A \cup\{x\}} \sum_{\substack{\sigma \in \mathfrak{S}_{A \cup\{x\}} \\
\text { s.t. } \sigma(y)=x}}\left(\sigma \tau_{x, y}\right)\left(\tau_{x, y} T\right) \\
& =\sum_{y \in A \cup\{x\}} \sum_{\tilde{\sigma} \in \mathfrak{S}_{A}} \tilde{\sigma}\left(\tau_{x, y} T\right) \\
& =\sum_{y \in A \cup\{x\}} G_{A}\left(\tau_{x, y} T\right)
\end{aligned}
$$

We now show that all Garnirs of minimal size are generated by Garnirs over a set consisting of a full row and a box below that row.

Lemma 16. Let $T \in \mathcal{T}_{\lambda, n}$. If $A \subset T_{0}$ of minimal size, then

$$
G_{A}(T) \in\left\langle G_{A^{\prime} \cup\left\{b_{0}\right\}}\left(T^{\prime}\right): T^{\prime} \in \mathcal{T}_{\lambda, n}, A^{\prime}=[b](r) \text { for some }[b](r) \subset T_{0}, b_{0}<[b](r)\right\rangle .
$$

Proof. Let $T \in \mathcal{T}_{\lambda, n}$ and $A \subset T_{0}$ such that $A \subset[b](r)$. Assume, without loss of generality, that $r \neq 1$ (else, replace $[b](r-1)$ in the following argument with $[b-1]\left(h_{b-1}\right)$ ). Let $B \subset T_{0}$ such that $B<[b](r), B \not \subset[b](r-1)$, and $|A \cup B|=w_{b}+1$. Assume $A \neq[b](r)$, i.e. $|B| \neq 1$. We will show that

$$
G_{A \cup B}(T) \in\left\langle G_{A^{\prime} \cup B^{\prime}}\left(T^{\prime}\right): T^{\prime} \in \mathcal{T}_{\lambda, n}, A^{\prime}=[b](r),\left|B^{\prime}\right|=1, B<A\right\rangle .
$$

Pick $x_{0} \in[b](r) \backslash A$ and $b_{0} \in B$.

$$
\begin{gathered}
\square \in A \\
\boxminus \in B \\
|A \cup B|=w_{b}+1
\end{gathered}
$$

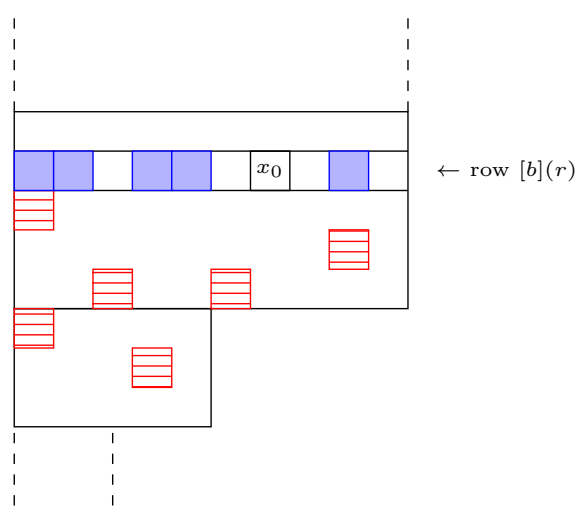

For all $x \in A \cup\left\{x_{0}\right\} \cup B$, define $\tau_{x_{0}, x}$ as before. Then for all $\sigma \in \mathfrak{S}_{A \cup\left\{x_{0}\right\} \cup B}$,

$$
\sigma\left(x_{0}\right)=x \Longleftrightarrow\left(\tau_{x_{0}, x} \sigma\right) x_{0}=x_{0} \Longleftrightarrow \tau_{x_{0}, x} \sigma \in \mathfrak{S}_{A \cup B}
$$

and

$$
\sigma\left(x_{0}\right)=x \Longleftrightarrow\left(\sigma \tau_{x_{0}, x}\right) x=x \Longleftrightarrow \sigma \tau_{x_{0}, x} \in \mathfrak{S}_{A \cup B \cup\left\{x_{0}\right\} \backslash\{x\}}
$$


Then,

$$
\begin{aligned}
& G_{A \cup\left\{x_{0}\right\} \cup B}(T)=\sum_{\sigma \in \mathfrak{S}_{A \cup\left\{x_{0}\right\} \cup B}} \sigma T \\
& =\sum_{a \in A \cup\left\{x_{0}\right\}} \sum_{\substack{\sigma \in \mathfrak{S}_{A \cup\left\{x_{0}\right\} \cup B}, \sigma\left(x_{0}\right)=a}} \sigma T+\sum_{b \in B} \sum_{\substack{\sigma \in \mathfrak{S}_{A \cup\left\{x_{0}\right\} \cup B}, \sigma\left(x_{0}\right)=b}} \sigma T \\
& =\sum_{a \in A \cup\left\{x_{0}\right\}} \sum_{\substack{\sigma \in \mathfrak{S}_{A \cup\left\{x_{0}\right\} \cup B}, \sigma\left(x_{0}\right)=a}} \tau_{x_{0}, a}\left(\tau_{x_{0}, a} \sigma\right) T+\sum_{b \in B} \sum_{\begin{array}{c}
\sigma \in \mathfrak{S}_{A \cup\left\{x_{0}\right\} \cup B}, \\
\sigma\left(x_{0}\right)=b
\end{array}}\left(\sigma \tau_{x_{0}, b}\right) \tau_{x_{0}, b} T
\end{aligned}
$$

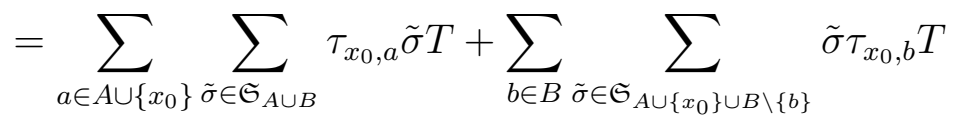

$$
\begin{aligned}
& =\sum_{a \in A \cup\left\{x_{0}\right\}} \tau_{x_{0}, a} G_{A \cup B}(T)+\sum_{b \in B} G_{A \cup\left\{x_{0}\right\} \cup B \backslash\{b\}}\left(\tau_{x_{0}, b} T\right)
\end{aligned}
$$

and as $\tau_{x_{0}, a}$ is a row permutation for all $a \in A \cup\left\{x_{0}\right\}$, up to row permutations we have

$$
G_{A \cup\left\{x_{0}\right\} \cup B}(T)=\left|A \cup\left\{x_{0}\right\}\right| G_{A \cup B}(T)+\sum_{b \in B} G_{A \cup\left\{x_{0}\right\} \cup B \backslash\{b\}}\left(\tau_{x_{0}, b} T\right) .
$$

Solving for $G_{A \cup B}(T)$ in the equation above we get

$$
G_{A \cup B}(T)=\frac{1}{\left|A \cup\left\{x_{0}\right\}\right|}\left(G_{A \cup\left\{x_{0}\right\} \cup B}(T)-\sum_{b \in B} G_{A \cup\left\{x_{0}\right\} \cup B \backslash\{b\}}\left(\tau_{x_{0}, b} T\right)\right) .
$$

By Lemma 15, $G_{A \cup B \cup\left\{x_{0}\right\}}(T)$ is generated by Garnirs over $A \cup\left\{x_{0}\right\} \cup B \backslash\left\{b_{0}\right\}$. Thus $G_{A \cup B}(T)$ is generated by Garnirs over $A^{\prime} \cup B^{\prime}$, where $A^{\prime}=A \cup\left\{x_{0}\right\}$, so that $\left|A^{\prime} \cap[b](r)\right|=$ $|A \cap[b](r)|+1$, and $B^{\prime}=B \backslash\{b\}$ for some $b \in B$, so that $\left|B^{\prime}\right|=|B|-1$. By induction, we get that

$$
G_{A \cup B}(T) \in\left\langle G_{A^{\prime} \cup B^{\prime}}\left(T^{\prime}\right): T^{\prime} \in \mathcal{T}_{\lambda, n}, A^{\prime}=[b](r),\left|B^{\prime}\right|=1\right\rangle .
$$

We now give a way to to write $G_{A \cup B}(T)$ as above (up to row permutation) as a sum of 2-cycles, which will make our calculations easier throughout.

Lemma 17. Let $A=[b](r)$ and $b_{0} \in T_{0} \backslash A$. Then for all $T \in \mathcal{T}_{\lambda, n}$, up to row permutation,

$$
G_{A \cup\left\{b_{0}\right\}}(T)=w_{b} ! \sum_{a \in A \cup\left\{b_{0}\right\}} \tau_{a, b_{0}} T .
$$

Proof.

$$
G_{A \cup\left\{b_{0}\right\}}(T)=\sum_{\sigma \in \mathfrak{S}_{A \cup\left\{b_{0}\right\}}} \sigma T
$$




$$
\begin{aligned}
& =\sum_{\tilde{\sigma} \in \mathfrak{S}_{A}} \sum_{a \in A \cup\left\{b_{0}\right\}} \tilde{\sigma} \tau_{a, b_{0}} T \\
& =w_{b} ! \sum_{a \in A \cup\left\{b_{0}\right\}} \tau_{a, b_{0}} T
\end{aligned}
$$

as all $\tilde{\sigma} \in \mathfrak{S}_{A}$ are row permutations and $|A|=w_{b}$.

\subsection{Completing the Proof of Theorem 14}

To prove Theorem 14, it remains to show that all Garnirs of the form $G_{A \cup B}(T)$ where $A=[b](r)$ and $|B|=1$, with $B<A$, are generated up to row permutation by Garnirs over hooks. We show that for any such $A$ and $B, G_{A \cup B}(T)$ is generated up to row permutation by Garnirs over $A^{\prime} \cup B^{\prime}$ where $A^{\prime}$ is a full row and $\left|B^{\prime}\right|=1$ with $B^{\prime}<A^{\prime}$, and where the distance between $A^{\prime}$ and $B^{\prime}$ is less than the distance between $A$ and $B$. Theorem 14 is then proved by iterating this until we get that $G_{A \cup B}(T)$ is generated up to row permutation by Garnirs over hooks.

Lemma 18. Let $T \in \mathcal{T}_{\lambda, n}, A=[b](r), B \subset T_{0}$ with $|B|=1$ and $B<A$. Then $G_{A \cup B}(T)$ is generated up to row permutation by $\left\{G_{A^{\prime}}\left(T^{\prime}\right) \mid T^{\prime} \in \mathcal{T}_{\lambda, n}, A^{\prime}\right.$ is a hook $\}$.

Proof. Let $A=[b](r)$ and $B=\left\{b_{0}\right\}$ with $b_{0} \in[c](s)$ and $[c](s)<[b](r)$. Let $j$ be the number of rows between $[b](r)$ and $[c](s)$. Without loss of generality we will assume that $r>j+1$ and $b_{0}=[b](r-j-1,1)$. Then, by Lemma 17, up to row permutation

$$
G_{A \cup B}(T)=w_{b} ! \sum_{a \in A \cup B} \tau_{a, b_{0}} T .
$$

We also have that for all $a \in A \cup B$, up to row permutation,

$$
G_{[b](r-j) \cup B}\left(\tau_{a, b_{0}} T\right)=w_{b} !\left(\tau_{a, b_{0}} T+\sum_{x \in[b](r-j)} \tau_{x, b_{0}} \tau_{a, b_{0}} T\right)
$$

and hence

$$
\tau_{a, b_{0}} T=\frac{1}{w_{b} !} G_{[b](r-j) \cup B}\left(\tau_{a, b_{0}} T\right)-\sum_{x \in[b](r-j)} \tau_{x, b_{0}} \tau_{a, b_{0}} T .
$$

Now observe that for all $a \in A \cup B$ and all $x \in[b](r-j), \tau_{x, b_{0}} \tau_{a, b_{0}} T=\tau_{a, x} \tau_{x, b_{0}} T$. Then,

$$
\begin{aligned}
G_{A \cup B}(T) & =w_{b} ! \sum_{a \in A \cup B} \tau_{a, b_{0}} T \\
& =w_{b} ! \sum_{a \in A \cup B}\left(\frac{1}{w_{b} !} G_{[b](r-j) \cup B}\left(\tau_{a, b_{0}} T\right)-\sum_{x \in[b](r-j)} \tau_{x, b_{0}} \tau_{a, b_{0}} T\right) \\
& =\sum_{a \in A \cup B} G_{[b](r-j) \cup B}\left(\tau_{a, b_{0}} T\right)-w_{b} ! \sum_{a \in A \cup B} \sum_{x \in[b](r-j)} \tau_{x, b_{0}} \tau_{a, b_{0}} T
\end{aligned}
$$




$$
\begin{aligned}
& =\sum_{a \in A \cup B} G_{[b](r-j) \cup B}\left(\tau_{a, b_{0}} T\right)-w_{b} ! \sum_{x \in[b](r-j)}\left(\tau_{x, b_{0}} T+\sum_{a \in A} \tau_{x, b_{0}} \tau_{a, b_{0}} T\right) \\
& =\sum_{a \in A \cup B} G_{[b](r-j) \cup B}\left(\tau_{a, b_{0}} T\right)-w_{b} ! \sum_{x \in[b](r-j)}\left(\tau_{x, b_{0}} T+\sum_{a \in A} \tau_{a, x} \tau_{x, b_{0}} T\right) \\
& =\sum_{a \in A \cup B} G_{[b](r-j) \cup B}\left(\tau_{a, b_{0}} T\right)-w_{b} ! \sum_{x \in[b](r-j)} G_{A \cup\{x\}}\left(\tau_{x, b_{0}} T\right) .
\end{aligned}
$$

So we have that for any $T \in \mathcal{T}_{\lambda, n}$ and any $A \subset T_{0}$ with $|A|>w_{A}, G_{A}(T)$ is generated up to row permutation by Garnir operators over hooks.

\subsection{Collapsing the Sum in the Image of a Pieri Inclusion Removing One Box}

The rest of Section 5 is devoted to collapsing the sum in the image $\Phi_{m}(T)$. We first consider the 1-path case, where the idea is that the sum over all possible paths between two boxes can be collapsed to a single tableau, modulo $\mathcal{R}_{\lambda \backslash X, n}$, with parity depending only on the number of rows between the two boxes. See Figure 2. We then generalize the result to 2-paths, before considering the $m$-path case.

Definition $19\left(\boldsymbol{\sigma}_{\boldsymbol{k}}^{\boldsymbol{A}}\right)$. Let $A \subset T_{0}$ be a hook with $[b](r)$ the top row of $A$. Label the boxes in $[b](r)$ as $a_{1}, \ldots, a_{w_{b}}$ and let $a_{0}$ be the box in $A$ below $[b](r)$. For $k=0, \ldots, w_{b}$, define $\sigma_{k}^{A}$ to be the permutation of $A$ that switches $a_{0}$ and $a_{k}$ and is the identity otherwise. For $T \in \mathcal{T}_{\lambda, n}$ and $0 \leqslant k \leqslant w_{b}$, let $A_{k}$ be the entry in the box $a_{k}$ and extend $\sigma_{k}^{A}$ to act on the entries of $T$, so that $\sigma_{k}^{A} A_{k}=A_{0}$ and $\sigma_{k}^{A}$ is the identity on $T$ otherwise. Then, by Lemma 17 , up to row permutation we have

$$
G_{A}(T)=w_{b} ! \sum_{k=0}^{w_{b}} \sigma_{k}^{A} T
$$

It will be useful to be able to identify those paths that are similar to a given $m$-path. Given an $m$-path $P$ and two rows $[b](r)$ and $[c](s)$, a $([b](r),[c](s))$-path extension of $P$ is an $m$-path $Q$ that is identical to $P$ except on the interval of rows $([b](r),[c](s))$ and on any boxes whose image under $P$ is in the interval of rows $([b](r),[c](s))$. In the row interval $([b](r),[c](s)), Q$ can differ from $P$, and in fact can even act on different boxes.

Example 20. Let the 1-path $P$ be given below.

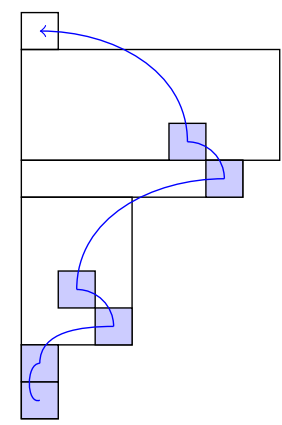



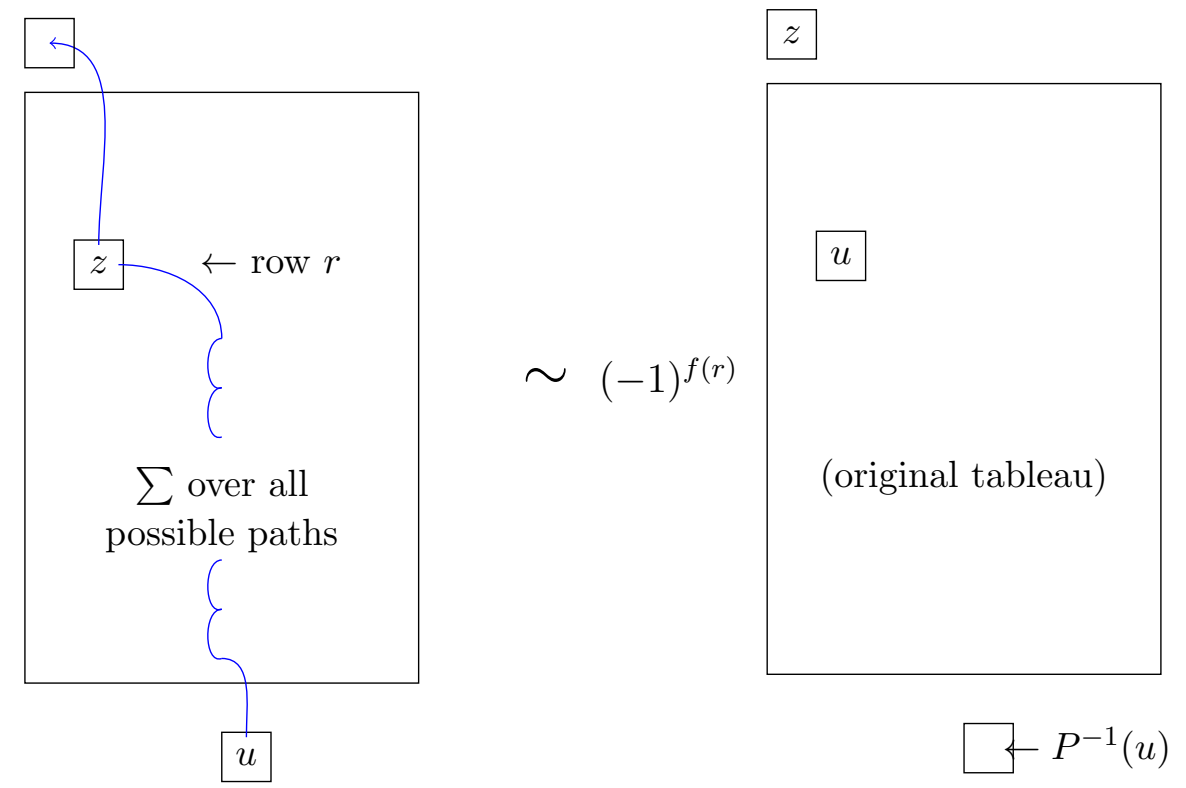

Figure 2: Collapsing a sum of paths.

For any $([2](2),[4](1))$-path extension $Q$ of $P$, it must be that $\{[1](1,1),[1](2,1)$, $[2](1,3)\} \subset R^{Q}$ as these are the boxes in $R^{P}$ outside of the interval of rows ([2](2), [4](1)). As $[2](1,3) \in P^{-1}(([2](2),[4](1))), Q$ must be identical to $P$ on $\{[1](1,1),\{[1](2,1)\}$, but it can be the case that $Q([2](1,3)) \neq P([2](1,3))$. Two such examples of $([2](2),[4](1))$-path extensions of $P$ are given below.
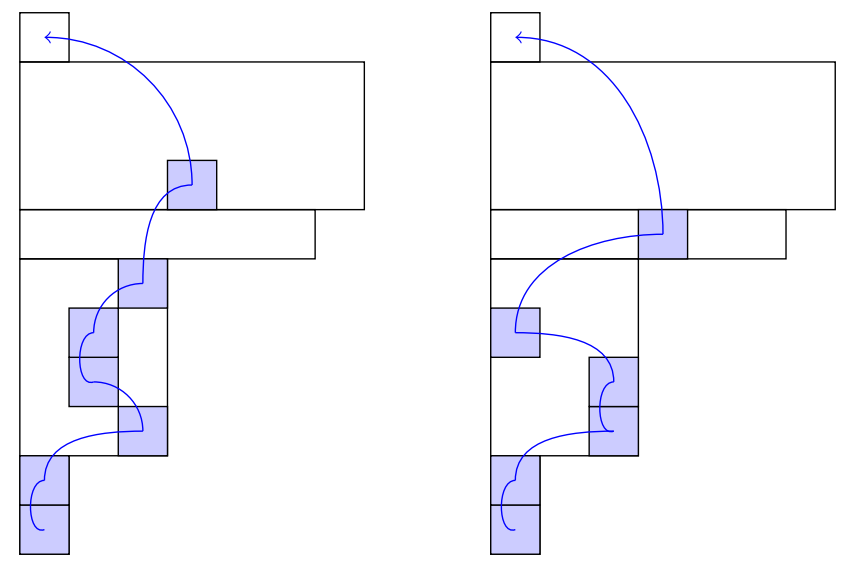
Now let the 2-path $P$ be given below.

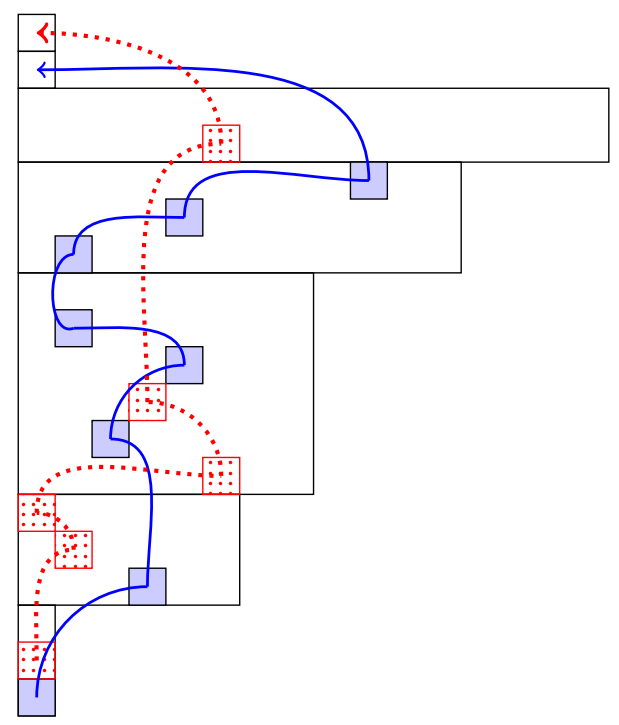

For any ([3](1), [4](1))-path extension $Q$ of $P$, it must be that $R^{Q} \backslash([3](1),[4](1))=$ $R^{P} \backslash([3](1),[4](1))$. As $\{[2](2,2),[2](3,1)\} \subset P^{-1}(([3](1),[4](1))), Q$ must be identical to $P$ on $R^{Q} \backslash(([3](1),[4](1)) \cup\{[2](2,2),[2](3,1)\})$, but $Q$ can differ from $P$ otherwise. An example of a $([3](1),[4](1))$-path extension of $P$ is given below.

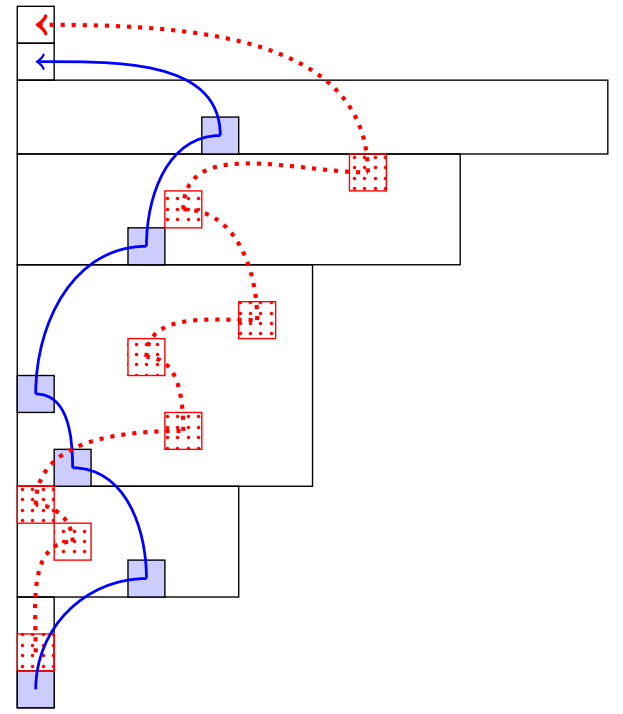

Given an evacuation route $R$ and a row $[b](r)$, define

$$
R_{<[b](r)}:=\{x \in R: x<[b](r)\} \quad \text { and } \quad R_{>[b](r)}:=\{x \in R:[b](r)<x\} .
$$

We formalize the notion of path extensions with the following definitions.

Definition 21 (Route Extension). Given an evacuation route $R$ and two rows $[b](r)$ and $[c](s)$ with $[b](r) \leqslant[c](s)$, an evacuation route $B$ is a $([b](r),[c](s))$-route extension of $R$ if $R_{<[b](r)}=B_{<[b](r)}$ and $R_{>[c](s)}=B_{>[c](s)}$. 
Definition 22 (Path Extension). Given an $m$-path $P$ and two rows $[b](r)$ and $[c](s)$ with $[b](r) \leqslant[c](s)$, an $m$-path $Q$ is a $([b](r),[c](s))$-path extension of $P$ if:

- $R^{Q}$ is a $([b](r),[c](s))$-route extension of $R^{P}$,

- $\left.P\right|_{R_{>[c](s)}^{P}}=\left.Q\right|_{R_{>[c](s)}^{P}}$

- $\left.P\right|_{R_{<[b](r)}^{P} \backslash I}=\left.Q\right|_{R_{<[b](r)}^{P} \backslash I}$, where $I=\left\{x \in R_{<[b](r)}^{P}: P(x) \in([b](r),[c](s))\right\}$.

For any $T \in \mathcal{T}_{\lambda, n}$, let

$$
X=\left\{x_{1}:=\left[b_{1}\right]\left(1, w_{b_{1}}\right)\right\} \text { and } Y=\left\{y_{1}:=[N+1](1,1)\right\}
$$

and let

$$
z_{1}:=T_{\left[b_{z}\right]\left(i_{1}, j_{1}\right)}
$$

for some $1 \leqslant b_{1} \leqslant b_{z} \leqslant N, 1 \leqslant i_{1} \leqslant h_{b_{z}}$, and $1 \leqslant j_{1} \leqslant w_{b_{z}}$. Let $u:=T_{\left[b_{u}\right]\left(i_{u}, j_{u}\right)}$ for some $b_{1} \leqslant b_{u} \leqslant b_{z}, 1 \leqslant i_{u} \leqslant h_{b_{u}}$, and $1 \leqslant j_{u} \leqslant w_{b_{u}}$, and let $P$ be any 1 -path on $\lambda$ removing $X$ such that $P\left(\left[b_{u}\right]\left(i_{u}, j_{u}\right)\right) \in\left[b_{z}\right](1)$ and $P\left(\left[b_{z}\right]\left(i_{1}, j_{1}\right)\right)>\left[b_{z}\right]\left(i_{1}\right)$, including the case $P\left(\left[b_{z}\right]\left(i_{1}, j_{1}\right)\right)=y_{1}$. Let

$$
\begin{aligned}
{[P]=} & \left\{1 \text {-paths } Q \text { on } \lambda: Q \text { is a }\left(\left[b_{z}\right](1),\left[b_{z}\right]\left(i_{1}\right)\right) \text {-path extension of } P\right. \\
& \text { with } \left.Q\left(\left[b_{z}\right]\left(i_{1}, j_{1}\right)\right)=P\left(\left[b_{z}\right]\left(i_{1}, j_{1}\right)\right)\right\}
\end{aligned}
$$

and $T^{\prime} \in \mathcal{T}_{\lambda \backslash X, n}$ be the unique tableau such that $T^{\prime}=T_{P}$ on $\lambda \backslash X$, where $T_{P}$ is defined as in Section 3.4 so that $\left(T_{P}\right)_{[b](i, j)}=T_{P^{-1}([b](i, j))}$, except on the interval of rows $\left(\left[b_{z}\right](1),\left[b_{z}\right]\left(i_{1}\right)\right)$, where $T^{\prime}=T$, except $T_{\left[b_{z}\right]\left(i_{1}, j_{1}\right)}^{\prime}=u$. We then have the following.

\section{Lemma 23.}

$$
\sum_{Q \in[P]} Q(T)=(-1)^{i_{1}-1} \alpha_{1}^{P} \otimes T^{\prime} \bmod V \otimes \mathcal{R}_{\lambda \backslash X, n} .
$$

Proof. Assume, without loss of generality, that $j_{1}=1$. We will show the case $b_{u}<b_{z}$, the case $b_{u}=b_{z}$ is similar. If $i_{1}=1$, then $[P]=\{P\}$, and so

$$
\begin{aligned}
\sum_{Q \in[P]} Q(T) & =P(T) \\
& =\alpha_{1}^{P} \otimes T^{\prime},
\end{aligned}
$$

as desired. Let $i_{1}=2$ and

$$
A=\left\{a_{1}:=\left[b_{z}\right](1, k), \ldots, a_{w_{b_{z}}}:=\left[b_{z}\right]\left(1, w_{b_{z}}\right)\right\} \cup\left\{a_{0}:=\left[b_{z}\right](2,1)\right\} .
$$

Then by Lemma 17 we have the following (see Figure 3).

$$
\sum_{Q \in[P]} Q(T)=\sum_{k=1}^{w_{b_{z}}} \alpha_{1}^{P} \otimes \sigma_{k}^{A} T^{\prime}
$$




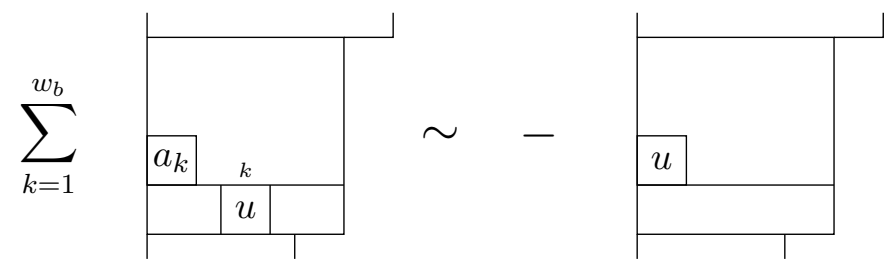

Figure 3: Collapsing a sum using Garnir operators.

$$
\begin{aligned}
& =\alpha_{1}^{P} \otimes\left(\frac{1}{w_{b_{z}} !} G_{A}\left(T^{\prime}\right)-T^{\prime}\right) \\
& =-\alpha_{1}^{P} \otimes T^{\prime} \bmod V \otimes \mathcal{R}_{\lambda \backslash X, n} .
\end{aligned}
$$

Now let $i_{1}>2$ and

$$
B=\left\{b_{1}:=\left[b_{z}\right]\left(i_{1}-1, k\right), \ldots, b_{w_{b_{z}}}:=\left[b_{z}\right]\left(i_{1}-1, w_{b_{z}}\right)\right\} \cup\left\{b_{0}:=\left[b_{z}\right]\left(i_{1}, 1\right)\right\} .
$$

Then by Lemma 17 and induction applied to each entry in $\left(i_{1}-1\right)^{b_{z}}$, we have

$$
\begin{aligned}
\sum_{Q \in[P]} Q(T) & =\sum_{k=1}^{w_{b_{z}}}(-1)^{i_{1}-2} \alpha_{1}^{P} \otimes \sigma_{k}^{B} T^{\prime} \\
& =(-1)^{i_{1}-2} \alpha_{1}^{P} \otimes\left(\frac{1}{w_{b_{z}} !} G_{B}\left(T^{\prime}\right)-T^{\prime}\right) \\
& =(-1)^{i_{1}-1} \alpha_{1}^{P} \otimes T^{\prime} \bmod V \otimes \mathcal{R}_{\lambda \backslash X, n} .
\end{aligned}
$$

Thus the claim holds for $1 \leqslant i_{1} \leqslant h_{b_{z}}$.

\subsection{Collapsing the Sum in the Image of a Pieri Inclusion Removing Many Boxes}

Lemma 23 also allows for calculations of sums of 2-paths, by applying the technique of the proof twice and "skipping" certain rows each time. That is, for any $T \in \mathcal{T}_{\lambda, n}$, let

$$
\begin{gathered}
X:=\left\{x_{1}:=\left[b_{1}\right]\left(1, w_{b_{1}}\right), x_{2}:=\left[b_{2}\right]\left(i_{2}, w_{b_{2}}\right)\right\} \\
Y:=\left\{y_{1}:=[N+1](1,1), y_{2}:=[N+1](2,1)\right\}
\end{gathered}
$$

and let

$$
z_{1}:=T_{\left[b_{z}\right]\left(i_{1}, j_{1}\right)}, z_{2}:=T_{\left[b_{z}\right]\left(i_{2}, j_{2}\right)}
$$

for some $1 \leqslant b_{1} \leqslant b_{z} \leqslant N, 1 \leqslant i_{2}<i_{1} \leqslant h_{b_{z}}$, and $1 \leqslant j_{1}, j_{2} \leqslant w_{b_{z}}$. Let

$$
u_{1}:=T_{\left[b_{u_{1}}\right]\left(i_{u_{1}}, j_{u_{1}}\right)}, u_{2}:=T_{\left[b_{u_{2}}\right]\left(i_{u_{2}}, j_{u_{2}}\right)}
$$


for some $b_{1} \leqslant b_{u_{1}}, b_{u_{2}} \leqslant b_{z}, 1 \leqslant i_{u_{1}} \leqslant h_{b_{u_{1}}}, 1 \leqslant j_{u_{1}} \leqslant w_{b_{u_{1}}}, 1 \leqslant i_{u_{2}} \leqslant h_{b_{u_{2}}}$, and $1 \leqslant j_{u_{2}} \leqslant w_{b_{u_{2}}}$. If $b_{u_{1}}=b_{u_{2}}$, then we also assume that $i_{u_{1}} \neq i_{u_{2}}$. Let $P$ be any 2-path on $\lambda$ such that

$$
\begin{gathered}
P\left(\left[b_{u_{1}}\right]\left(i_{u_{1}}, j_{u_{1}}\right)\right) \in\left[b_{z}\right](1), P\left(\left[b_{u_{2}}\right]\left(i_{u_{2}}, j_{u_{2}}\right)\right) \in\left[b_{z}\right](2), \\
P\left(\left[b_{z}\right]\left(i_{1}, j_{1}\right)\right), P\left(\left[b_{z}\right]\left(i_{2}, j_{2}\right)\right)>\left[b_{z}\right]\left(i_{1}\right) .
\end{gathered}
$$

Assume, without loss of generality, that $P\left(\left[b_{z}\right]\left(i_{1}, j_{1}\right)\right), P\left(\left[b_{z}\right]\left(i_{2}, j_{2}\right)\right) \notin Y$. Let

$$
\begin{aligned}
{[P]=} & \left\{2 \text {-paths } Q \text { on } \lambda: Q \text { is a }\left(\left[b_{z}\right](1),\left[b_{z}\right]\left(i_{1}\right)\right) \text {-path extension of } P\right. \\
& \text { with } \left.Q\left(\left[b_{z}\right]\left(i_{1}, j_{1}\right)\right)=P\left(\left[b_{z}\right]\left(i_{1}, j_{1}\right)\right), Q\left(\left[b_{z}\right]\left(i_{2}, j_{2}\right)\right)=P\left(\left[b_{z}\right]\left(i_{2}, j_{2}\right)\right)\right\}
\end{aligned}
$$

and $T^{\prime} \in \mathcal{T}_{\lambda \backslash X, n}$ be the unique tableau such that $T^{\prime}=T_{P}$ on $(\lambda \backslash X)$, except on the interval of rows $\left(\left[b_{z}\right](1),\left[b_{z}\right]\left(i_{1}\right)\right)$, where $T^{\prime}=T$, except $T_{\left[b_{z}\right]\left(i_{1}, j_{1}\right)}^{\prime}=u, T_{\left[b_{z}\right]\left(i_{2}, j_{2}\right)}^{\prime}=v$. We then have the following.

\section{Corollary 24.}

$$
\sum_{Q \in[P]} Q(T)=(-1)^{i_{1}-2+i_{2}-2} \frac{\alpha_{2}^{P}}{\alpha_{1}^{P}} \otimes T^{\prime} \bmod \bigwedge^{2} V \otimes \mathcal{R}_{\lambda \backslash X, n} .
$$

Proof. Apply the techniques from the proof of Lemma 23 to get a sum of tableaux with $u_{1}$ in the box $\left[b_{z}\right]\left(i_{1}, j_{1}\right)$, skipping row $\left[b_{z}\right]\left(i_{2}\right)$, then apply the techniques again to get $u_{2}$ in the box $\left[b_{z}\right]\left(i_{2}, j_{2}\right)$, skipping row $\left[b_{z}\right]\left(i_{2}-1\right)$.

The same technique used above immediately generalizes to sums of $m$-path extensions. Fix $m>2$. For any $T \in \mathcal{T}_{\lambda, n}$, let

$$
X=\left\{x_{1}=\left[b_{1}\right]\left(1, w_{b_{1}}\right), \ldots, x_{m}=\left[b_{m}\right]\left(i_{m}, w_{b_{m}}\right)\right\}
$$

be a removal set and

$$
z_{k}=T_{\left[b_{z}\right]\left(i_{k}, j_{k}\right)} \quad \text { for } 1 \leqslant k \leqslant m
$$

for some $1 \leqslant b_{1} \leqslant b_{z} \leqslant N, 1 \leqslant i_{m}<\cdots<i_{1} \leqslant h_{b_{z}}$, and $1 \leqslant j_{k} \leqslant w_{b_{z}}$ for $1 \leqslant k \leqslant m$. Let

$$
u_{k}=T_{\left[b_{u_{k}}\right]\left(i_{u_{k}}, j_{u_{k}}\right)} \quad \text { for } 1 \leqslant k \leqslant m
$$

for some $b_{1} \leqslant b_{u_{k}} \leqslant b_{z}, 1 \leqslant i_{u_{k}} \leqslant h_{b_{u_{k}}}, 1 \leqslant j_{u_{k}} \leqslant w_{b_{u_{k}}}$. If $b_{u_{k}}=b_{u_{l}}$ for $k \neq l$, then we also assume that $i_{u_{k}} \neq i_{u_{l}}$. Let $P$ be any $m$-path on $\lambda$ such that, for $1 \leqslant k \leqslant m$,

$$
P\left(\left[b_{u_{k}}\right]\left(i_{u_{k}}, j_{u_{k}}\right)\right) \in\left[b_{z}\right](k)
$$

and

$$
P\left(\left[b_{z}\right]\left(i_{k}, j_{k}\right)\right)>\left[b_{z}\right]\left(i_{1}\right) .
$$

Assume, without loss of generality, that $P\left(\left[b_{z}\right]\left(i_{k}, j_{k}\right)\right) \notin Y$ for $1 \leqslant k \leqslant m$. Let

$$
[P]=\left\{m \text {-paths } Q \text { on } \lambda: Q \text { is a }\left(\left[b_{z}\right](1),\left[b_{z}\right]\left(i_{1}\right)\right) \text {-path extension of } P\right.
$$
such that $Q\left(\left[b_{z}\right]\left(i_{k}, j_{k}\right)\right)=P\left(\left[b_{z}\right]\left(i_{k}, j_{k}\right)\right)$ for $\left.1 \leqslant k \leqslant m\right\}$

and $T^{\prime} \in \mathcal{T}_{\lambda \backslash X, n}$ be the unique tableau such that $T^{\prime}=T_{P}$ on $(\lambda \backslash X)$ except on the interval of rows $\left(\left[b_{z}\right](1),\left[b_{z}\right]\left(i_{2}\right)\right)$, where $T^{\prime}=T$ except $T_{\left[b_{z}\right]\left(i_{k}, j_{k}\right)}^{\prime}=u_{k}$ for $1 \leqslant k \leqslant m$. We then have the following. 
Corollary 25.

$$
\sum_{Q \in[P]} Q(T)=(-1)^{i_{1}-m+\cdots+i_{m}-m} \begin{array}{c|c}
\alpha_{m}^{P} \\
\\
\hline \alpha_{1}^{P}
\end{array} \otimes T^{\prime} \bmod \bigwedge^{m} V \otimes \mathcal{R}_{\lambda \backslash X, n}
$$

Proof. Assume, without loss of generality, that

$$
\begin{aligned}
i_{1} & >i_{2} \\
i_{2} & >i_{3}+1 \\
\vdots & \\
i_{m-1} & >i_{m}+(m-1)-1 .
\end{aligned}
$$

Otherwise, the following goes through by skipping the appropriate rows. Apply the techniques from the proof of Lemma 23 to get a sum of tableaux with $u_{1}$ in the box $\left[b_{z}\right]\left(i_{1}, j_{1}\right)$, skipping rows $i_{m}^{z}, \ldots, i_{2}^{z}$. Then iterate the techniques again to get $u_{k}$ in the box $\left[b_{z}\right]\left(i_{k}, j_{k}\right)$, skipping rows $\left[b_{z}\right]\left(i_{m}\right), \ldots,\left[b_{z}\right]\left(i_{k+1}\right)$ and rows $\left[b_{z}\right]\left(i_{k}-1\right), \ldots\left[b_{z}\right]\left(i_{k}-(k-1)\right)$.

\section{The Pieri Inclusion Removing One Box is a GL(V)-map}

\subsection{Stating the Theorem and Set-Up}

For all of Section 6 , fix $X=\left\{x_{1}:=\left[b_{1}\right]\left(1, w_{b_{1}}\right)\right\} \subset T_{0}$ to be removed. Let

$$
\Phi_{1}: \mathcal{F}_{\lambda, n} \rightarrow V \otimes \mathcal{F}_{\lambda \backslash X, n}
$$

be as in Section 3.4.

Theorem 26. $\Phi_{1}$ is a $G L(V)$-map, i.e. $\Phi_{1}$ descends to

$$
\Phi_{1}: \mathbb{S}_{\lambda}(V) \rightarrow V \otimes \mathbb{S}_{\lambda \backslash X}(V)
$$

and $\Phi_{1}$ is $G L(V)$-equivariant.

We will show that this map is $\mathfrak{g l}(V)$-equivariant, which implies $G L(V)$-equivariance. Identifying $G L(V)$ with $G L_{n}(\mathbb{C})$, the standard Cartan subalgebra of $\mathfrak{g l}(V)=\mathfrak{g l}_{n}(\mathbb{C})$ is the set of all diagonal matrices (see, for example, $[5, \S 8]$ ). For each simple root vector $\alpha_{i}$ with respect this standard Cartan subalgebra, the action of $e_{\alpha_{i}}$ on a tableau $T$ generates a sum of tableau $\widetilde{T}$ where each entry $i$ in $T$ is replaced by an $i+1$. Similarly, for each $e_{-\alpha_{i}}$, where each entry $i$ in $T$ is replaced by an $i-1$. As $\Phi_{1}$ is a sum over 1-paths that move entries up the diagram, acting with $e_{\alpha_{i}}$ and applying $\Phi_{1}$ to the sum is the same as acting in the opposite order. As the simple root vectors generate $\mathfrak{g l}(V), \Phi_{1}$ is $\mathfrak{g l} l(V)$-equivariant.

To prove Theorem 26, it remains to show that

$$
\Phi_{1}\left(\mathcal{R}_{\lambda, n}\right) \subset V \otimes \mathcal{R}_{\lambda \backslash X, n}
$$


It is clear that $\Phi_{1}$ preserves Property 1 as it is a sum over all 1-paths, and hence we must show that Property 2 holds, i.e. for all $T \in \mathcal{T}_{\lambda, n}$ and all $A \subset T_{0}$ with $|A|>w_{A}$,

$$
\Phi_{1}\left(G_{A}(T)\right) \in V \otimes \mathcal{R}_{\lambda \backslash X, n} .
$$

By Theorem 14, it is enough to show that the displayed inclusion (3) holds for all hooks $A$. If $A$ is a hook, either $A$ is completely contained in a block $b$, with $1 \leqslant b \leqslant N$, or $A$ is contained in two blocks, $b$ and $b+1$, with $1 \leqslant b \leqslant N-1$. We consider these two options separately.

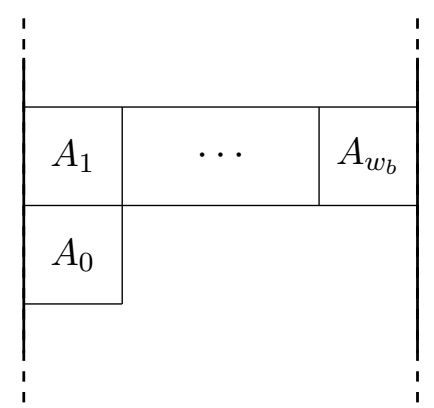

$A \subset[b]$.

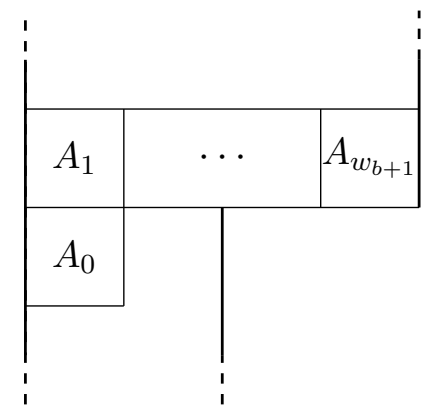

$A \subset[b] \cup[b+1]$.

\subsection{Showing Theorem 26 Holds for Hooks Contained in a Single Block}

We first show that the inclusion (3) holds for all hooks $A \subset[b]$, for some $1 \leqslant b \leqslant N$. For the rest of Section 6.2, fix $T \in \mathcal{T}_{\lambda, n}$ and

$$
A=\left\{a_{0}:=[b]\left(i_{0}, 1\right), a_{1}:=[b]\left(i_{0}+1,1\right), \ldots, a_{w_{b}}:=[b]\left(i_{0}+1, w_{b}\right)\right\} \subset T_{0}
$$

with $1 \leqslant i_{0}<h_{b}$, so that $A \subset[b]$. Denote the entries of $A$ in $T$ by $A_{k}=T_{a_{k}}$ for $k=0,1, \ldots, w_{b}$. Then by Lemma 17 , up to row permutation we have

$$
\Phi_{1}\left(G_{A}(T)\right)=\sum_{P} \frac{(-1)^{P}}{H(P)} P\left(\sum_{\sigma \in \mathfrak{S}_{A}} \sigma T\right)=w_{b} ! \sum_{P} \sum_{k=0}^{w_{b}} \frac{(-1)^{P}}{H(P)} P\left(\sigma_{k}^{A} T\right),
$$

where the sum is over all 1-paths $P$ on $\lambda$ removing $X$. The set of all $P\left(\sigma_{k}^{A} T\right)$ appearing in the image $\Phi_{1}\left(G_{A}(T)\right)$ above is the union of the following disjoint sets.

The $P\left(\sigma_{k}^{A} T\right)$ that miss $A$,

$$
\mathcal{T}_{1}=\left\{P\left(\sigma_{k}^{A} T\right): R^{P} \cap A=\emptyset\right\} .
$$

The $P\left(\sigma_{k}^{A} T\right)$ that hit $A$ and keep $A$ in block $b$,

$$
\mathcal{T}_{2}=\left\{P\left(\sigma_{k}^{A} T\right): R^{P} \cap A \neq \emptyset, P(A) \leqslant[b]\right\} .
$$

The $P\left(\sigma_{k}^{A} T\right)$ that hit $A$ and move the entry $A_{i}$ above block $b$, including $P\left(\sigma_{k}^{A} A_{i}\right) \in Y$,

$$
\mathcal{T}_{3}=\bigsqcup_{i=0}^{w_{b}} \mathcal{T}_{3}^{i}, \quad \text { where } \quad \mathcal{T}_{3}^{i}=\left\{P\left(\sigma_{k}^{A} T\right): R^{P} \cap A \neq \emptyset, P\left(\sigma_{k}^{A} A_{i}\right)>[b]\right\}
$$


We can then write the image up to row permutation as

$$
\Phi_{1}\left(G_{A}(T)\right)=w_{b} ! \sum_{P} \sum_{k=0}^{w_{b}} \frac{(-1)^{P}}{H(P)} P\left(\sigma_{k}^{A} T\right)=w_{b} ! \sum_{j=1}^{3} \sum_{P\left(\sigma_{k}^{A} T\right) \in \mathcal{T}_{j}} \frac{(-1)^{P}}{H(P)} P\left(\sigma_{k}^{A} T\right) .
$$

We show that for $j=1,2$, and 3 ,

$$
\sum_{P\left(\sigma_{k}^{A} T\right) \in \mathcal{T}_{j}} \frac{(-1)^{P}}{H(P)} P\left(\sigma_{k}^{A} T\right) \in V \otimes \mathcal{R}_{\lambda \backslash X, n},
$$

and hence the inclusion (3) holds for all hooks $A$ with $A \subset[b]$.

Case 27. In this case we show that the sum over all paths that miss $A$ is in $V \otimes \mathcal{R}_{\lambda \backslash X, n}$, i.e.

$$
\sum_{P\left(\sigma_{k}^{A} T\right) \in \mathcal{T}_{1}} \frac{(-1)^{P}}{H(P)} P\left(\sigma_{k}^{A} T\right) \in V \otimes \mathcal{R}_{\lambda \backslash X, n} .
$$

Proof. As $P$ misses $A$ for all $P\left(\sigma_{k}^{A} T\right) \in \mathcal{T}_{1},\left.P\right|_{A}=\mathrm{id}_{A}$, and thus

$$
P\left(\sigma_{k}^{A} T\right)=\alpha_{1}^{P} \otimes \sigma_{k}^{A} T_{P} \text { for all } 0 \leqslant k \leqslant w_{b} .
$$

So by Lemma $17, \bmod V \otimes \mathcal{R}_{\lambda \backslash X, n}$,

$$
\begin{aligned}
\sum_{P\left(\sigma_{k}^{A} T\right) \in \mathcal{T}_{1}} \frac{(-1)^{P}}{H(P)} P\left(\sigma_{k}^{A} T\right) & =\sum_{P\left(\sigma_{0}^{A} T\right) \in \mathcal{T}_{1}} \sum_{k=0}^{w_{b}} \frac{(-1)^{P}}{H(P)} P\left(\sigma_{k}^{A} T\right) \\
& =\sum_{P\left(\sigma_{0}^{A} T\right) \in \mathcal{T}_{1}} \sum_{k=0}^{w_{b}} \frac{(-1)^{P}}{H(P)} \alpha_{1}^{P} \otimes \sigma_{k}^{A} T_{P} \\
& =\sum_{P\left(\sigma_{0}^{A} T\right) \in \mathcal{T}_{1}} \frac{1}{w_{b} !} \frac{(-1)^{P}}{H(P)} \alpha_{1}^{P} \otimes G_{A}\left(T_{P}\right) \\
& =0 .
\end{aligned}
$$

Case 28. In this case we show that the sum over all paths that hit $A$ and keep $A$ in block $b$ is in $V \otimes \mathcal{R}_{\lambda \backslash X, n}$, i.e.

$$
\sum_{P\left(\sigma_{k}^{A} T\right) \in \mathcal{T}_{2}} \frac{(-1)^{P}}{H(P)} P\left(\sigma_{k}^{A} T\right) \in V \otimes \mathcal{R}_{\lambda \backslash X, n}
$$

Proof. For a 1-path $P$, let $P^{-1}$ be the unique map of boxes

$$
P^{-1}: \lambda \cup Y \rightarrow \lambda \cup Y
$$


such that for all $x \in \lambda \cup Y, P^{-1}(P(x))=x$. For all $k=0,1, \ldots, w_{b}$, let

$$
\tau_{k}^{A}:=P \sigma_{k}^{A} P^{-1} \in \mathfrak{S}_{P(A)},
$$

so that $\tau_{k}^{A}$ permutes $P\left(a_{0}\right)$ and $P\left(a_{k}\right)$ and is the identity otherwise. Extend $\tau_{k}^{A}$ to act on the entries of $T_{P}$. Then, $\bmod V \otimes \mathcal{R}_{\lambda \backslash X, n}$,

$$
\begin{aligned}
\sum_{P\left(\sigma_{k}^{A} T\right) \in \mathcal{T}_{2}} \frac{(-1)^{P}}{H(P)} P\left(\sigma_{k}^{A} T\right) & =\sum_{P\left(\sigma_{0}^{A} T\right) \in \mathcal{T}_{2}} \sum_{k=0}^{w_{b}} \frac{(-1)^{P}}{H(P)} P\left(\sigma_{k}^{A} T\right) \\
& =\sum_{P\left(\sigma_{0}^{A} T\right) \in \mathcal{T}_{2}} \sum_{k=0}^{w_{b}} \frac{(-1)^{P}}{H(P)} P\left(\sigma_{k}^{A} P^{-1}(P(T))\right) \\
& =\sum_{P\left(\sigma_{0}^{A} T\right) \in \mathcal{T}_{2}} \sum_{k=0}^{w_{b}} \frac{(-1)^{P}}{H(P)} \alpha_{1}^{P} \otimes \tau_{k}^{A} T_{P} .
\end{aligned}
$$

As $P(A) \subset[b]$ and $|P(A)|=w_{b}+1$, by the proof of Lemma 17 we have, $\bmod V \otimes \mathcal{R}_{\lambda \backslash X, n}$,

$$
\sum_{P\left(\sigma_{0}^{A} T\right) \in \mathcal{T}_{2}} \sum_{k=0}^{w_{b}} \frac{(-1)^{P}}{H(P)} \alpha_{1}^{P} \otimes \tau_{k}^{A} T_{P}=\sum_{P\left(\sigma_{0}^{A} T\right) \in \mathcal{T}_{2}} \frac{1}{w_{b} !} \frac{(-1)^{P}}{H(P)} \alpha_{1}^{P} \otimes G_{P(A)} T_{P}=0 .
$$

Remark 29. Notice that the proofs of Case 27 and Case 28 did not depend on removing a single box nor on $A$ being contained in a single block, and so these will generalize to the $m \geqslant 1$ case for both options for a hook $A$.

Case 30. In this case we show that the sum over all paths that hit $A$ and move the entry $A_{i}$ above block $b$ is in $V \otimes \mathcal{R}_{\lambda \backslash X, n}$. We will assume that $b>b_{1}$, as the case $b \leqslant b_{1}$ can be treated similarly. It is enough to show that for each $i=0, \ldots, w_{b}$,

$$
\sum_{P\left(\sigma_{k}^{A} T\right) \in \mathcal{T}_{3}^{i}} \frac{(-1)^{P}}{H(P)} P\left(\sigma_{k}^{A} T\right) \in V \otimes \mathcal{R}_{\lambda \backslash X, n} .
$$

We will show the case $i=0$, with the cases $i=1, \ldots, w_{b}$ being similar.

Proof. For the rest of Case 30 let $\mathcal{T}:=\mathcal{T}_{3}^{0}$ and, for any 1-path $P$, let $\tilde{h}^{P}:=h^{P}-h_{b}^{P}$. Define the relation $\sim$ on $\mathcal{T}$ by

$$
P\left(\sigma_{k}^{A} T\right) \sim Q\left(\sigma_{j}^{A} T\right) \Longleftrightarrow Q \text { is a }\left([b](1),[b]\left(i_{0}+1\right)\right) \text {-path extension of } P .
$$

It is clear that this defines an equivalence relation on $\mathcal{T}$, so that

$$
\sum_{P\left(\sigma_{k}^{A} T\right) \in \mathcal{T}} \frac{(-1)^{P}}{H(P)} P\left(\sigma_{k}^{A} T\right)=\sum_{\left[P\left(\sigma_{k}^{A} T\right)\right] \in \mathcal{T} / \sim} \sum_{Q\left(\sigma_{k}^{A} T\right) \in\left[P\left(\sigma_{k}^{A} T\right)\right]} \frac{(-1)^{Q}}{H(Q)} Q\left(\sigma_{k}^{A} T\right) .
$$

Pick $P\left(\sigma_{0}^{A} T\right) \in \mathcal{T}$ with $[b](i, 1) \in R^{P}$ for all $i=1, \ldots, i_{0}$, and let $\left[b_{u}\right]\left(i_{u}, j_{u}\right)=$ $P^{-1}([b](1,1))$, with $u:=T_{\left[b_{u}\right]\left(i_{u}, j_{u}\right)}$, as pictured below. 


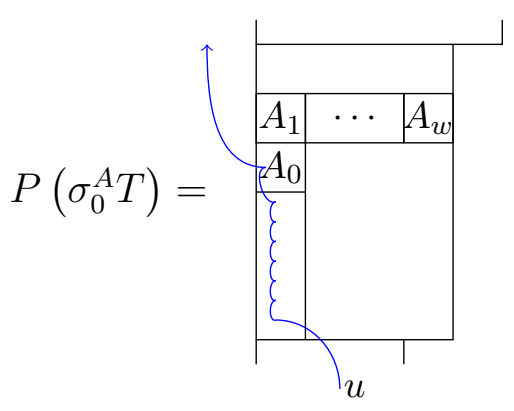

It is then enough to show that

$$
\sum_{Q\left(\sigma_{k}^{A} T\right) \in\left[P\left(\sigma_{0}^{A} T\right)\right]} \frac{(-1)^{Q}}{H(Q)} Q\left(\sigma_{k}^{A} T\right) \in V \otimes \mathcal{R}_{\lambda \backslash X, n} .
$$

In fact, as $\tilde{h^{Q}}=\tilde{h^{P}}$ and $H(Q)=H(P)$ for all $Q\left(\sigma_{k}^{A} T\right) \in\left[P\left(\sigma_{0}^{A} T\right)\right]$, it is enough to show that

$$
\sum_{Q\left(\sigma_{k}^{A} T\right) \in\left[P\left(\sigma_{0}^{A} T\right)\right]}(-1)^{h_{b}^{Q}} Q\left(\sigma_{k}^{A} T\right) \in V \otimes \mathcal{R}_{\lambda \backslash X, n} .
$$

Observe that $\left[P\left(\sigma_{0}^{A} T\right)\right]$ can be written as the disjoint union

$$
\left[P\left(\sigma_{0}^{A} T\right)\right]=\bigsqcup_{i=1}^{3}\left[P\left(\sigma_{0}^{A} T\right)\right]_{i},
$$

where the $\left[P\left(\sigma_{0}^{A} T\right)\right]_{i}$ are defined as follows.

The paths acting on $\sigma_{0}^{A} T$,

$$
\left[P\left(\sigma_{0}^{A} T\right)\right]_{1}=\left\{Q\left(\sigma_{0}^{A} T\right) \in\left[P\left(\sigma_{0}^{A} T\right)\right]\right\}
$$

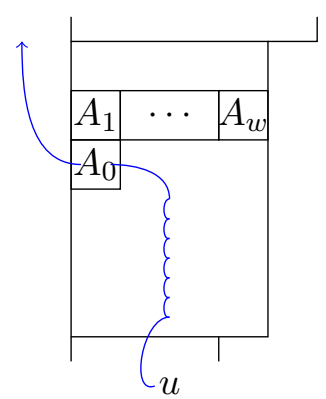

the paths acting on $\sigma_{k}^{A} T, k \neq 0$, that hit $a_{0}=\sigma_{k}^{a} a_{k}$,

$$
\left[P\left(\sigma_{0}^{A} T\right)\right]_{2}=\left\{Q\left(\sigma_{k}^{A} T\right) \in\left[P\left(\sigma_{0}^{A} T\right)\right]: k \neq 0, a_{0} \in R^{Q}\right\},
$$




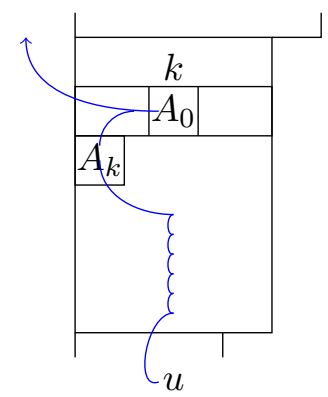

and the paths acting on $\sigma_{k}^{A} T, k \neq 0$, that miss $a_{0}=\sigma_{k}^{a} a_{k}$,

$$
\left[P\left(\sigma_{0}^{A} T\right)\right]_{3}=\left\{Q\left(\sigma_{k}^{A} T\right) \in\left[P\left(\sigma_{0}^{A} T\right)\right]: k \neq 0, a_{0} \notin R^{Q}\right\} .
$$

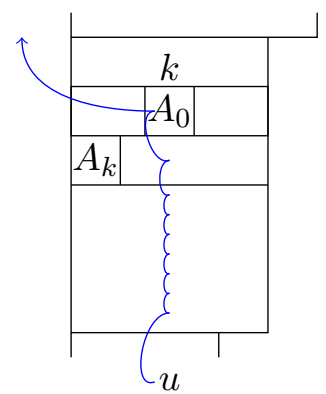

Let $T^{\prime} \in \mathcal{T}_{\lambda \backslash X}$ be the unique tableau with $T^{\prime}=T_{P}$ on $(\lambda \backslash X) \backslash[b]$ and $T^{\prime}=T$ on $[b]$ except $T_{a_{0}}^{\prime}=u$, as shown below.

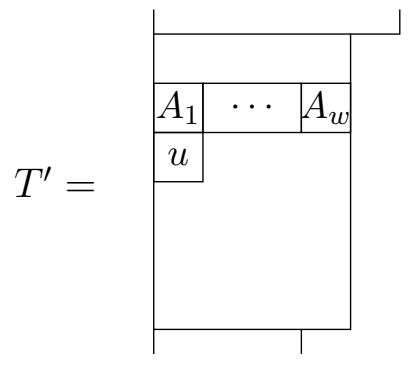

Then by Lemma 23 and applications of $G_{A}$ we have, $\bmod V \otimes \mathcal{R}_{\lambda \backslash X, n}$,

$$
\begin{gathered}
\sum_{Q\left(\sigma_{0}^{A} T\right) \in\left[P\left(\sigma_{0}^{A} T\right)\right]_{1}}(-1)^{h_{b}^{Q}} Q\left(\sigma_{0}^{A} T\right)=(-1)^{i_{0}+i_{0}-1} \alpha_{1}^{P} \otimes T^{\prime}=-\alpha_{1}^{P} \otimes T^{\prime}, \\
\sum_{Q\left(\sigma_{k}^{A} T\right) \in\left[P\left(\sigma_{0}^{A} T\right)\right]_{2}}(-1)^{h_{b}^{Q}} Q\left(\sigma_{0}^{A} T\right)=(-1)^{i_{0}+1+i_{0}-1} w_{b} \alpha_{1}^{P} \otimes T^{\prime}=w_{b} \alpha_{1}^{P} \otimes T^{\prime},
\end{gathered}
$$

and

$$
\sum_{Q\left(\sigma_{k}^{A} T\right) \in\left[P\left(\sigma_{0}^{A} T\right)\right]_{3}}(-1)^{h_{b}^{Q}} Q\left(\sigma_{k}^{A} T\right)=(-1)^{i_{0}+1+1+i_{0}-1}\left(w_{b}-1\right) \alpha_{1}^{P} \otimes T^{\prime}
$$




$$
=-\left(w_{b}-1\right) \alpha_{1}^{P} \otimes T^{\prime}
$$

Now as

$$
\sum_{Q\left(\sigma_{k}^{A} T\right) \in\left[P\left(\sigma_{0}^{A} T\right)\right]}(-1)^{h_{b}^{Q}} Q\left(\sigma_{k}^{A} T\right)=\sum_{i=1}^{3} \sum_{Q\left(\sigma_{k}^{A} T\right) \in\left[P\left(\sigma_{0}^{A} T\right)\right]_{i}}(-1)^{h_{b}^{Q}} Q\left(\sigma_{k}^{A} T\right),
$$

$\bmod V \otimes \mathcal{R}_{\lambda \backslash X, n}$

$$
\sum_{Q\left(\sigma_{k}^{A} T\right) \in\left[P\left(\sigma_{0}^{A} T\right)\right]}(-1)^{h_{b}^{Q}} Q\left(\sigma_{k}^{A} T\right)=\left(-1+w_{b}-w_{b}+1\right) \alpha_{1}^{P} \otimes T^{\prime}=0 .
$$

\subsection{Showing Theorem 26 Holds for Hooks Intersecting Two Blocks}

We now show that the inclusion (3) holds for all hooks $A \subset[b] \cup[b+1]$ for some $1 \leqslant b \leqslant$ $N-1$. For the rest of Section 6.3, fix $T \in \mathcal{T}_{\lambda, n}$ and

$$
A=\left\{a_{0}:=[b]\left(h_{b}, 1\right), a_{1}:=[b+1](1,1), \ldots, a_{w_{b+1}}:=[b+1]\left(1, w_{b+1}\right)\right\} \subset T_{0},
$$

so that $A \subset[b] \cup[b+1]$. Denote the entries of $A$ in $T$ by $A_{k}=T_{a_{k}}$ for $k=0,1, \ldots, w_{b+1}$.

Then, by Lemma 17, up to row permutation we have

$$
\Phi_{1}\left(G_{A}(T)\right)=\sum_{P} \frac{(-1)^{P}}{H(P)} P\left(\sum_{\sigma \in \mathfrak{S}_{A}} \sigma T\right)=w_{b+1} ! \sum_{P} \sum_{k=0}^{w_{b+1}} \frac{(-1)^{P}}{H(P)} P\left(\sigma_{k}^{A} T\right),
$$

where the sum is over all 1-paths $P$ on $\lambda$ removing $X$. The set of all $P\left(\sigma_{k}^{A} T\right)$ appearing in the image $\Phi_{1}\left(G_{A}(T)\right)$ above is the union of the following disjoint sets.

The $P\left(\sigma_{k}^{A} T\right)$ that miss $A$,

$$
\mathcal{T}_{1}=\left\{P\left(\sigma_{k}^{A} T\right): R^{P} \cap A=\emptyset\right\} .
$$

The $P\left(\sigma_{k}^{A} T\right)$ that hit $A$ and keep $A$ in blocks $b$ and $b+1$,

$$
\mathcal{T}_{2}=\left\{P\left(\sigma_{k}^{A} T\right): R^{P} \cap A \neq \emptyset, P(A) \leqslant[b+1]\right\} .
$$

The $P\left(\sigma_{k}^{A} T\right)$ that hit $A$ and move the entry $A_{i}$ above block $b+1$, including $P\left(\sigma_{k}^{A} A_{i}\right) \in Y$,

$$
\mathcal{T}_{3}=\bigsqcup_{i=0}^{w_{b+1}} \mathcal{T}_{3}^{i} \quad \text { where } \quad \mathcal{T}_{3}^{i}=\left\{P\left(\sigma_{k}^{A} T\right) \in \mathcal{T}_{3}: R^{P} \cap A \neq \emptyset, P\left(\sigma_{k}^{A} A_{i}\right)>[b+1]\right\} .
$$

Then we have, up to row permutation,

$$
\Phi_{1}\left(G_{A}(T)\right)=w_{b+1} ! \sum_{P} \sum_{k=0}^{w_{b+1}} \frac{(-1)^{P}}{H(P)} P\left(\sigma_{k}^{A} T\right)=w_{b+1} ! \sum_{j=1}^{3} \sum_{P\left(\sigma_{k}^{A} T\right) \in \mathcal{T}_{j}} \frac{(-1)^{P}}{H(P)} P\left(\sigma_{k}^{A} T\right) .
$$


We show that for $j=1,2$, and 3 ,

$$
\sum_{P\left(\sigma_{k}^{A} T\right) \in T_{j}} \frac{(-1)^{P}}{H(P)} P\left(\sigma_{k}^{A} T\right) \in V \otimes \mathcal{R}_{\lambda \backslash X, n},
$$

and hence the inclusion (3) holds for all hooks $A \subset[b] \cup[b+1]$.

The inclusion above with $j=1$ follows from the proof of Case 27 and the inclusion above with $j=2$ follows from the proof of Case 28. It remains to show that the above inclusion holds for $j=3$.

Case 31. In this case we show that the sum over all paths that hit $A$ and move the entry $A_{i}$ above block $b+1$ is in $V \otimes \mathcal{R}_{\lambda \backslash X, n}$. It is enough to show that for $i=0, \ldots, w_{b+1}$,

$$
\sum_{P\left(\sigma_{k}^{A} T\right) \in \mathcal{T}_{3}^{i}} \frac{(-1)^{P}}{H(P)} P\left(\sigma_{k}^{A} T\right) \in V \otimes \mathcal{R}_{\lambda \backslash X, n} .
$$

We will show the case $i=0$, with the cases $i=1, \ldots, w_{b+1}$ being similar.

Proof. Note that as we are considering paths that hit $A$ in this case, we must have that $b \geqslant b_{1}-1$. We will show the case $b>b_{1}-1$, as the case $b=b_{1}-1$ (and hence $a_{w_{b+1}}=x_{1}$ ) uses similar techniques combined with the techniques in Cases 27 and 28.

For the rest of Case 31 , let $\mathcal{T}:=\mathcal{T}_{3}^{0}$ and, for any 1-path $P$, define $\tilde{h}^{P}:=h^{P}-h_{b}^{P}$ and

$$
\tilde{H(P)}=\frac{H(P)}{H_{b}(P) H_{b+1}(P)} .
$$

Define the relation $\sim$ on $\mathcal{T}$ by

$$
P\left(\sigma_{k}^{A} T\right) \sim Q\left(\sigma_{j}^{A} T\right) \Longleftrightarrow Q \text { is a }([b](1),[b+1](1)) \text {-path extension of } P .
$$

It is clear that this defines an equivalence relation on $\mathcal{T}$, so that

$$
\sum_{P\left(\sigma_{k}^{A} T\right) \in \mathcal{T}} \frac{(-1)^{P}}{H(P)} P\left(\sigma_{k}^{A} T\right)=\sum_{\left[P\left(\sigma_{k}^{A} T\right)\right] \in \mathcal{T} / \sim} \sum_{!\left(\sigma_{k}^{A} T\right) \in\left[P\left(\sigma_{k}^{A} T\right)\right]} \frac{(-1)^{Q}}{H(Q)} Q\left(\sigma_{k}^{A} T\right) .
$$

Pick $P\left(\sigma_{0}^{A} T\right) \in \mathcal{T}$ with $[b](i, 1) \in R^{P}$ for all $i=1, \ldots, h_{b}$, and let $\left[b_{u}\right]\left(i_{u}, j_{u}\right)=$ $P^{-1}([b](1,1))$ with $u:=T_{\left[b_{u}\right]\left(i_{u}, j_{u}\right)}$, as illustrated below.

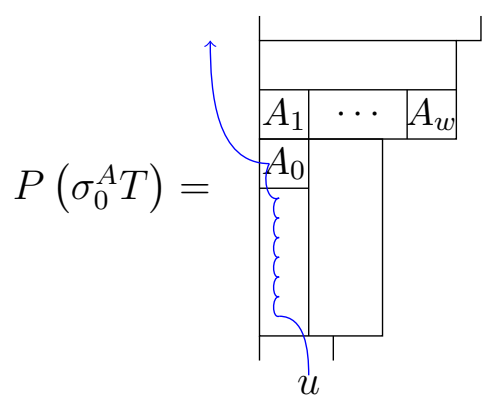


It is then enough to show that

$$
\sum_{Q\left(\sigma_{k}^{A} T\right) \in\left[P\left(\sigma_{0}^{A} T\right)\right]} \frac{(-1)^{Q}}{H(Q)} Q\left(\sigma_{k}^{A} T\right) \in V \otimes \mathcal{R}_{\lambda \backslash X, n} .
$$

In fact, as $\tilde{h^{Q}}=\tilde{h^{P}}$ and $\tilde{H(Q)}=\tilde{H(P)}$ for all $Q\left(\sigma_{k}^{A} T\right) \in\left[P\left(\sigma_{0}^{A} T\right)\right]$, it is enough to show that

$$
\sum_{!\left(\sigma_{k}^{A} T\right) \in\left[P\left(\sigma_{0}^{A} T\right)\right]} \frac{(-1)^{h_{b}^{Q}}}{H_{b}(Q) H_{b+1}(Q)} Q\left(\sigma_{k}^{A} T\right) \in V \otimes \mathcal{R}_{\lambda \backslash X, n} .
$$

Observe that $\left[P\left(\sigma_{0}^{A} T\right)\right]$ can be written as the disjoint union

$$
\mathcal{T}=\bigsqcup_{i=1}^{6}\left[P\left(\sigma_{0}^{A} T\right)\right]_{i}
$$

where the $\left[P\left(\sigma_{0}^{A} T\right)\right]_{i}$ are defined as follows.

The paths acting on $\sigma_{0}^{A} T$,

$$
\left[P\left(\sigma_{0}^{A} T\right)\right]_{1}=\left\{Q\left(\sigma_{0}^{A} T\right) \in\left[P\left(\sigma_{0}^{A} T\right)\right]\right\}
$$

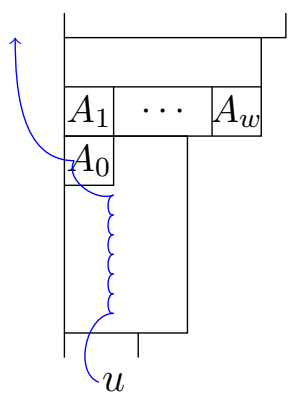

The paths acting on $\sigma_{k}^{A} T, k \neq 0$, that miss block $b$,

$$
\left[P\left(\sigma_{0}^{A} T\right)\right]_{2}=\left\{Q\left(\sigma_{k}^{A} T\right) \in\left[P\left(\sigma_{0}^{A} T\right)\right]: k \neq 0, R^{Q} \cap[b]=\emptyset\right\},
$$

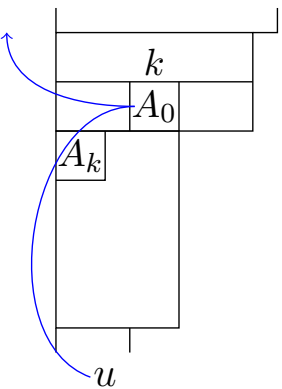

the paths acting on $\sigma_{k}^{A} T, k \neq 0$, that hit $a_{0}=\sigma_{k}^{a} a_{k}$,

$$
\left[P\left(\sigma_{0}^{A} T\right)\right]_{3}=\left\{Q\left(\sigma_{k}^{A} T\right) \in\left[P\left(\sigma_{0}^{A} T\right)\right]: k \neq 0, a_{0} \in R^{Q}\right\},
$$




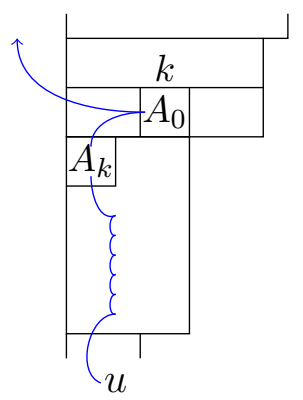

the paths acting on $\sigma_{k}^{A} T, k \neq 0$, that miss $a_{0}=\sigma_{k}^{a} a_{k}$ but hit row $[b]\left(h_{b}\right)$,

$$
\left[P\left(\sigma_{0}^{A} T\right)\right]_{4}=\left\{Q\left(\sigma_{k}^{A} T\right) \in\left[P\left(\sigma_{0}^{A} T\right)\right]: k \neq 0,[b]\left(h_{b}, j\right) \in R^{Q} \text { for some } 2 \leqslant j \leqslant w_{b}\right\},
$$

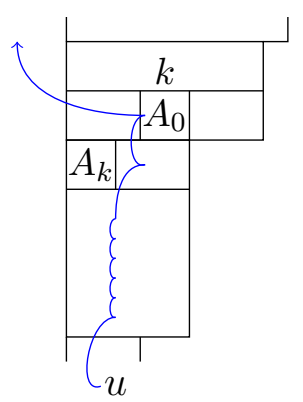

the paths acting on $\sigma_{k}^{A} T, k \neq 0$, that miss row $[b]\left(h_{b}\right)$ and leave block $b$ from an odd row,

$$
\begin{aligned}
{\left[P\left(\sigma_{0}^{A} T\right)\right]_{5}=\left\{Q\left(\sigma_{k}^{A} T\right)\right.} & \in\left[P\left(\sigma_{0}^{A} T\right)\right]: k \neq 0, Q([b](i, j))=a_{k} \\
& \text { for some } \left.1 \leqslant j \leqslant w_{b}, 1 \leqslant i<h_{b}, i \text { odd }\right\}
\end{aligned}
$$

and the paths acting on $\sigma_{k}^{A} T, k \neq 0$, that miss row $[b]\left(h_{b}\right)$ and leave block $b$ from an even row,

$$
\begin{aligned}
{\left[P\left(\sigma_{0}^{A} T\right)\right]_{6}=\left\{Q\left(\sigma_{k}^{A} T\right)\right.} & \in\left[P\left(\sigma_{0}^{A} T\right)\right]: k \neq 0, Q([b](i, j))=a_{k} \\
& \text { for some } \left.1 \leqslant j \leqslant w_{b}, 1 \leqslant i<h_{b}, i \text { even }\right\} .
\end{aligned}
$$

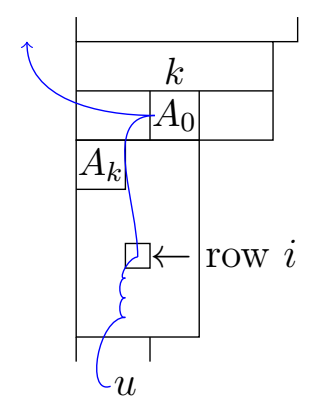

Let $T^{\prime} \in \mathcal{T}_{\lambda \backslash X, n}$ be the unique tableau with $T^{\prime}=T_{P}$ on $(\lambda \backslash X) \backslash([b] \cup[b+1])$ and $T^{\prime}=T$ on $[b] \cup[b+1]$ except $T_{a_{0}}^{\prime}=u$, as shown below. 


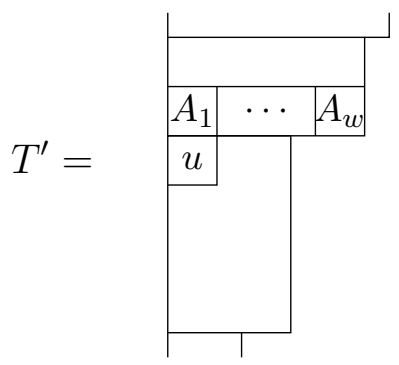

By Lemma 23 and applications of $G_{A}$ we have, $\bmod V \otimes \mathcal{R}_{\lambda \backslash X, n}$,

$$
\begin{aligned}
& \sum_{Q\left(\sigma_{0}^{A} T\right) \in\left[P\left(\sigma_{0}^{A} T\right)\right]_{1}}(-1)^{h_{b}^{Q}} Q\left(\sigma_{0}^{A} T\right)=\frac{(-1)^{h_{b}+h_{b}-1}}{H(b)} \alpha_{1}^{P} \otimes T^{\prime} \\
& =\frac{-H(b+1)}{H(b) H(b+1)} \alpha_{1}^{P} \otimes T^{\prime}, \\
& \sum_{Q\left(\sigma_{k}^{A} T\right) \in\left[P\left(\sigma_{0}^{A} T\right)\right]_{2}}(-1)^{h_{b}^{Q}} Q\left(\sigma_{k}^{A} T\right)=\frac{(-1)^{1+1}}{H(b+1)} \alpha_{1}^{P} \otimes T^{\prime} \\
& =\frac{H(b)}{H(b) H(b+1)} \alpha_{1}^{P} \otimes T^{\prime}, \\
& \sum_{Q\left(\sigma_{k}^{A} T\right) \in\left[P\left(\sigma_{0}^{A} T\right)\right]_{3}}(-1)^{h_{b}^{Q}} Q\left(\sigma_{k}^{A} T\right)=\frac{(-1)^{h_{b}+1+h_{b}-1} w_{b+1}}{H(b) H(b+1)} \alpha_{1}^{P} \otimes T^{\prime} \\
& =\frac{w_{b+1}}{H(b) H(b+1)} \alpha_{1}^{P} \otimes T^{\prime}, \\
& \sum_{Q\left(\sigma_{k}^{A} T\right) \in\left[P\left(\sigma_{0}^{A} T\right)\right]_{4}}(-1)^{h_{b}^{Q}} Q\left(\sigma_{k}^{A} T\right)=\frac{(-1)^{h_{b}+1+1+h_{b}-1}\left(w_{b}-1\right)}{H(b) H(b+1)} \alpha_{1}^{P} \otimes T^{\prime} \\
& =\frac{1-w_{b}}{H(b) H(b+1)} \alpha_{1}^{P} \otimes T^{\prime}, \\
& \sum_{Q\left(\sigma_{k}^{A} T\right) \in\left[P\left(\sigma_{0}^{A} T\right)\right]_{5}}(-1)^{h_{b}^{Q}} Q\left(\sigma_{k}^{A} T\right)=\sum_{\substack{1 \leqslant i<h_{b} \\
i \text { odd }}} \frac{(-1)^{i+1+1+i-1+1}}{H(b) H(b+1)} \alpha_{1}^{P} \otimes T^{\prime}
\end{aligned}
$$




$$
=\sum_{\substack{1 \leqslant i<h_{b} \\ i \text { odd }}} \frac{1}{H(b) H(b+1)} \alpha_{1}^{P} \otimes T^{\prime}
$$

and

$$
\begin{aligned}
\sum_{Q\left(\sigma_{k}^{A} T\right) \in\left[P\left(\sigma_{0}^{A} T\right)\right]_{5}}(-1)^{h_{b}^{Q}} Q\left(\sigma_{k}^{A} T\right) & =\sum_{\substack{1 \leqslant i<h_{b} \\
i \text { even }}} \frac{(-1)^{i+1+1+i-1+1}}{H(b) H(b+1)} \alpha_{1}^{P} \otimes T^{\prime} \\
& =\sum_{\substack{1 \leqslant i<h_{b} \\
i \text { even }}} \frac{1}{H(b) H(b+1)} \alpha_{1}^{P} \otimes T^{\prime} .
\end{aligned}
$$

Then as $H(b+1)=H(b)+w_{b+1}-w_{b}+h_{b}$ and

$$
\begin{gathered}
\sum_{\substack{1 \leqslant i<h_{b} \\
i \text { odd }}} \frac{1}{H(b) H(b+1)} \alpha_{1}^{P} \otimes T^{\prime}+\sum_{\substack{1 \leqslant i<h_{b} \\
i \text { even }}} \frac{1}{H(b) H(b+1)} \alpha_{1}^{P} \otimes T^{\prime} \\
=\frac{h_{b}-1}{H(b) H(b+1)} \alpha_{1}^{P} \otimes T^{\prime}
\end{gathered}
$$

we get, $\bmod V \otimes \mathcal{R}_{\lambda \backslash X, n}$,

$$
\begin{aligned}
\sum_{Q\left(\sigma_{k}^{A} T\right) \in\left[P\left(\sigma_{0}^{A} T\right)\right]}(-1)^{h_{b}^{Q}} Q\left(\sigma_{k}^{A} T\right) & =\sum_{i=1, \ldots, 6} \sum_{Q\left(\sigma_{k}^{A} T\right) \in\left[P\left(\sigma_{0}^{A} T\right)\right]_{i}}(-1)^{h_{b}^{Q}} Q\left(\sigma_{k}^{A} T\right) \\
& =\frac{-H(b+1)+H(b)+w_{b+1}+1-w_{b}+h_{b}-1}{H(b) H(b+1)} \alpha_{1}^{P} \otimes T^{\prime} \\
& =0 .
\end{aligned}
$$

Thus the inclusion (3) holds for all hooks $A \subset[b] \cup[b+1]$, and so Theorem 26 holds.

\section{The Pieri Inclusion Removing Many Boxes is a GL(V)-map}

\subsection{Stating the Theorem and Set-Up}

For all of Section 7, fix a removal set $X=\left\{x_{1}=\left[b_{1}\right]\left(1, w_{b_{1}}\right), \ldots, x_{m}=\left[b_{m}\right]\left(i_{m}, w_{b_{m}}\right)\right\} \subset \lambda$. Let

$$
\Phi_{m}: \mathcal{F}_{\lambda, n} \rightarrow \bigwedge^{m} V \otimes \mathcal{F}_{\lambda \backslash X, n}
$$

be as in Section 4.3.

Theorem 32. $\Phi_{m}$ is a $G L(V)$-map, i.e. $\Phi_{m}$ descends to

$$
\Phi_{m}: \mathbb{S}_{\lambda}(V) \rightarrow \bigwedge^{m} V \otimes \mathbb{S}_{\lambda \backslash X}(V)
$$

and $\Phi_{m}$ is $G L(V)$-equivariant. 
As before, it is clear that $\Phi_{m}$ is $\mathfrak{g l}(V)$-equivariant, and hence $G L(V)$-equivariant, by construction. To prove Theorem 32, it remains to show that

$$
\Phi_{m}\left(\mathcal{R}_{\lambda, n}\right) \subset \bigwedge^{m} V \otimes \mathcal{R}_{\lambda \backslash X, n}
$$

It is clear that $\Phi_{m}$ preserves Property 1 as it is a sum over all $m$-paths, and hence it remains to be shown that Property 2 holds, i.e. for all $T \in \mathcal{T}_{\lambda, n}$ and all $A \subset T_{0}$ with $|A|>w_{A}$,

$$
\Phi_{m}\left(G_{A}(T)\right) \in \bigwedge^{m} V \otimes \mathcal{R}_{\lambda \backslash X, n}
$$

\subsection{Outlining the Proof of Theorem 32 in the Two Box Removal Case}

The proof that the displayed inclusion (4) holds for $m=2$ is similar in technique to the proofs in Sections 6.2 and 6.3 that the inclusion (3) holds, and so we will outline the proof but omit the details.

Following the techniques from Section 6 , to show that

$$
\begin{aligned}
\Phi_{2}\left(G_{A}(T)\right) & =\sum_{P} \frac{(-1)^{P}}{H(P)} P\left(\sum_{\sigma \in \mathfrak{S}_{A}} \sigma T\right) \\
& =w_{b} ! \sum_{P} \sum_{k=0}^{w_{b}} \frac{(-1)^{P}}{H(P)} P\left(\sigma_{k}^{A} T\right) \in \bigwedge^{2} V \otimes \mathcal{R}_{\lambda \backslash X, n}
\end{aligned}
$$

for some hook $A$ with top row in block [b], where the sum is over all 2-paths $P$ removing $X$, we first identify the set of all $P\left(\sigma_{k}^{A} T\right)$ appearing in the image $\Phi_{2}\left(G_{A}(T)\right)$ as the union of the following disjoint sets. We use the same notation for the boxes and entries in the hook $A$ as was used in Sections 6.2 and 6.3.

The $P\left(\sigma_{k}^{A} T\right)$ that miss $A$,

$$
\mathcal{T}_{1}=\left\{P\left(\sigma_{k}^{A} T\right): R^{P} \cap A=\emptyset\right\} .
$$

The $P\left(\sigma_{k}^{A} T\right)$ that hit $A$ and keep $A$ in block $b$,

$$
\mathcal{T}_{2}=\left\{P\left(\sigma_{k}^{A} T\right): R^{P} \cap A \neq \emptyset, P(A) \subset[b]\right\} .
$$

The $P\left(\sigma_{k}^{A} T\right)$ that have exactly one orbit in $[b]$ and move $A_{i}$ above $[b]$,

$$
\mathcal{T}_{3}=\bigsqcup_{0 \leqslant i \leqslant w_{b}} \mathcal{T}_{3}^{i}
$$

where

$$
\mathcal{T}_{3}^{i}=\left\{P\left(\sigma_{k}^{A} T\right): \text { exactly one of } R_{1}, R_{2} \text { intersects }[b] \text { and } P\left(\sigma_{k} A_{i}\right)>[b]\right\}
$$


The $P\left(\sigma_{k}^{A} T\right)$ that move $A_{i}$ and $A_{j}$ above $[b]$,

$$
\mathcal{T}_{4}=\bigsqcup_{0 \leqslant i<j \leqslant w_{b}} \mathcal{T}_{4}^{i, j}
$$

where

$$
\mathcal{T}_{4}^{i, j}=\left\{P\left(\sigma_{k}^{A} T\right) \in \mathcal{T}_{4}: P\left(\sigma_{k}^{A} a_{i}\right)>[b] \text { and } P\left(\sigma_{k}^{A} a_{j}\right)>[b]\right\} .
$$

The $P\left(\sigma_{k}^{A} T\right)$ that move $A_{i}$ and a box $z \in[b]$, with $z<A$, above $[b]$,

$$
\mathcal{T}_{5}=\bigsqcup_{\substack{0 \leqslant i \leqslant w_{b}, z=[b]\left(i_{z}, j_{z}\right), 1 \leqslant i_{z} \leqslant i_{0}-1,1 \leqslant j_{z} \leqslant w_{b}}} \mathcal{T}_{5}^{i, z}
$$

where

$$
\mathcal{T}_{5}^{i, z}=\left\{P\left(\sigma_{k}^{A} T\right) \in \mathcal{T}_{5}: P\left(\sigma_{k}^{A} a_{i}\right)>[b], P(z)>[b]\right\} .
$$

The $P\left(\sigma_{k}^{A} T\right)$ that move $A_{i}$ and a box $z \notin A$ in row $i_{0}$ above [b],

$$
\mathcal{T}_{6}=\bigsqcup_{0 \leqslant i \leqslant w_{b}, 2 \leqslant j \leqslant w_{b}} \mathcal{T}_{6}^{i, j}
$$

where

$$
\mathcal{T}_{6}^{i, j}=\left\{P\left(\sigma_{k}^{A} T\right) \in \mathcal{T}_{6}: P\left(\sigma_{k}^{A} a_{i}\right)>[b], P\left([b]\left(i_{0}, j\right)\right)>[b]\right\} .
$$

The $P\left(\sigma_{k}^{A} T\right)$ that move $A_{i}$ and a box in [b] above $A$ above $[b]$,

$$
\mathcal{T}_{7}=\bigsqcup_{\substack{0 \leqslant i \leqslant w_{b} \\ 1 \leqslant j \leqslant w_{b}}} \mathcal{T}_{7}^{i, j}
$$

where

$$
\mathcal{T}_{7}^{i, j}=\left\{P\left(\sigma_{k}^{A} T\right) \in \mathcal{T}_{7}: P\left(\sigma_{k}^{A} A_{i}\right)>[b],[b]\left(i_{0}+2, j\right) \in R^{P}\right\} .
$$

Then, up to row permutation,

$$
\Phi_{2}\left(G_{A}(T)\right)=w_{b} ! \sum_{P} \sum_{k=0}^{w_{b}} \frac{(-1)^{P}}{H(P)} P\left(\sigma_{k}^{A} T\right)=w_{b} ! \sum_{j=1, \ldots, 7} \sum_{P\left(\sigma_{k}^{A} T\right) \in \mathcal{T}_{j}} \frac{(-1)^{P}}{H(P)} P\left(\sigma_{k}^{A} T\right)
$$

By applying the tools in Section 5 along with the definitions in Section 4 (with the noted difference from before of keeping track of the sign for the terms in the alternating product), one can show that for $j=1, \ldots, 7$,

$$
\sum_{P\left(\sigma_{k}^{A} T\right) \in T_{j}} \frac{(-1)^{P}}{H(P)} P\left(\sigma_{k}^{A} T\right) \in \bigwedge^{2} V \otimes \mathcal{R}_{\lambda \backslash X, n},
$$

and hence the inclusion (4) holds when $m=2$. 


\subsection{Showing the General Case from the Two Box Case}

Proving that the inclusion (4) holds for general $m$ can be done using identical techniques to the $m=2$ case, and so has also been omitted. To illustrate why the general case reduces to the $m=2$ case, recall that by Theorem 14 it is enough to show that the inclusion (4) holds only for hooks, and that hooks consist of boxes in exactly two rows. So, a given hook can only intersect at most two orbits of any $m$-path. Showing the general case then comes down to the $m=2$ case by considering each pair of orbits separately and "skipping" the rows of the corresponding evacuation route that do not intersect the given hook, as illustrated in the figures below.
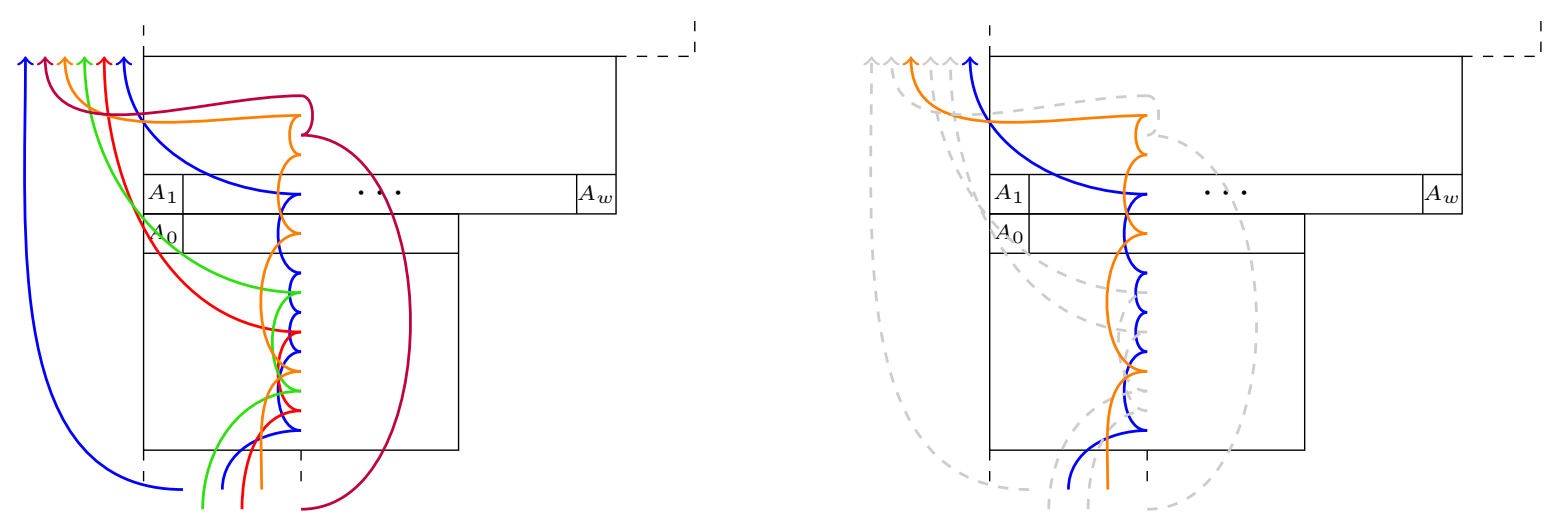

After using the techniques outlined above to show that the inclusion (4) holds for all $m$, we then have that Theorem 32 holds for all $m$.

\section{Relating Pieri Inclusion Descriptions}

In this section we show that our description of the Pieri inclusion removing one box is the negative of the previously known description and explicitly describe how the iteration of our description of the Pieri inclusion removing one box is related to our description of the Pieri inclusion removing many boxes. Finally, we show that our description of the Pieri inclusion removing many boxes also describes the symmetric case.

Let $\Phi_{1}$ be Pieri inclusion constructed in Section 3 and $\widetilde{\Phi}_{1}$ be the Pieri inclusion given by Olver in [10] (for a more recent explicit description of Olver's map see [12]).

Theorem 33. For $\widetilde{\Phi}_{1}$ and $\Phi_{1}$ as above,

$$
\Phi_{1}=-\widetilde{\Phi}_{1}
$$

Proof. Let $T_{\lambda} \in \mathcal{T}_{\lambda, n}$ and $T_{\lambda \backslash X} \in \mathcal{T}_{\lambda \backslash X, n}$ be the diagrams corresponding to highest weight vectors as in Section 2. Then, in the image of $T_{\lambda}$, the coefficient of

$$
\alpha \otimes T_{\lambda \backslash X}
$$


where

$$
\alpha=\sum_{i=b_{1}}^{N} h_{i}
$$

is readily seen to be $-w_{b_{1}}$ in the image of $\Phi$ and $w_{b_{1}}$ in the image of $\widetilde{\Phi}_{1}$. By uniqueness of the Pieri inclusion up to scalar multiple (Schur's Lemma), we then have

$$
\Phi=-\widetilde{\Phi}_{1}
$$

Given removal set $X=\left\{x_{1}=\left[b_{1}\right]\left(1, w_{b_{1}}\right), \ldots, x_{m}=\left[b_{m}\right]\left(i_{m}, w_{b_{m}}\right)\right\} \subset \lambda$, let $\Phi_{1}^{m}$ be the map given by composing the one box removal map where the column of removed boxes is extended by one each time, i.e. $\Phi_{1}^{1}=\Phi_{1}$ and for $1 \leqslant k \leqslant m-1$

$$
\Phi_{1}^{k+1}(T)=\sum_{P} \frac{(-1)^{P}}{H(P)} P\left(\Phi_{1}^{k}(T)\right)
$$

where the sum is over all 1-paths $P$ on $\lambda \backslash\left\{x_{1}, \ldots, x_{k}\right\}$ removing $x_{k+1}$ and

$$
P\left(\begin{array}{c|c}
\alpha_{k}^{Q} \\
\hline \vdots \\
\hline \alpha_{1}^{Q}
\end{array} \otimes T_{Q}\right)=\begin{gathered}
\alpha_{1}^{P} \\
\hline \alpha_{k}^{Q} \\
\vdots \vdots \\
\hline \alpha_{1}^{Q}
\end{gathered} \otimes\left(T_{Q}\right)_{P}
$$

and $\alpha_{1}^{P}$ is the entry removed by $P$ from $T_{Q}$.

Lemma 34. $\Phi_{1}^{m}$ is a $G L(V)$-map.

Proof. By the previous theorem this follows from the proof in [14, Corollary 1.8], where it is shown for the iteration of Olver's map.

Let

$$
\Phi_{m}: \mathbb{S}_{\lambda}(V) \rightarrow \bigwedge^{m} V \otimes \mathbb{S}_{\lambda \backslash X}(V)
$$

be the Pieri inclusion constructed in Section 4.

Theorem 35. For $\Phi_{1}^{m}$ and $\Phi_{m}$ as above,

$$
\Phi_{1}^{m}=m ! \cdot \Phi_{m} .
$$

Proof. Let $T_{\lambda} \in \mathcal{T}_{\lambda, n}$ and $T_{\lambda \backslash X} \in \mathcal{T}_{\lambda \backslash X, n}$ be the diagrams corresponding to highest weight vectors as in Section 2. Then in the image of $T_{\lambda}$, the coefficient of

$$
\begin{array}{|c|c|c|}
\hline \alpha_{m} & \\
\hline \vdots & \otimes T_{\lambda \backslash X} \\
\hline \alpha_{1} &
\end{array}
$$


where

$$
\alpha_{1}=\sum_{i=b_{1}}^{N} h_{i}
$$

and

$$
\alpha_{k}=\alpha_{1}-(k-1)
$$

for $2 \leqslant k \leqslant m$ is readily seen to be $(-1)^{m} w_{b_{1}}^{m}$ in the image of $\Phi_{m}$ and $(-1)^{m} w_{b_{1}}^{m} m$ ! in the image of $\Phi_{1}^{m}$. By uniqueness of the Pieri inclusion up to scalar multiple, we then have

$$
\Phi_{1}^{m}=m ! \cdot \Phi_{m}
$$

Define the map

$$
\Phi_{m}^{\prime}: \mathbb{S}_{\lambda}(V) \rightarrow S^{m}(V) \otimes \mathbb{S}_{\lambda \backslash X}(V)
$$

just as we have defined $\Phi_{m}$ in Section 4 except for redefining, for all $m$-paths $P$ on $\lambda$ removing $X$,

$$
P(T)=e_{\alpha_{1}^{P}} \cdots e_{\alpha_{m}^{P}} \otimes T_{P} \in S^{m}(V) \otimes \mathbb{S}_{\lambda \backslash X}(V) .
$$

Theorem 36. The map

$$
\Phi_{m}^{\prime}: \mathbb{S}_{\lambda}(V) \rightarrow S^{m}(V) \otimes \mathbb{S}_{\lambda \backslash X}(V)
$$

is a $G L(V)$-map.

Proof. As $\Phi_{m}$ is a $G L(V)$-map, similar to [14, Corollary 1.8], this follows by the results of Sections 6 and 7 by keeping track of a sign.

\section{The Image of a Highest Weight Vector and Computational Complexity}

\subsection{An Optimal Description of a Highest Weight Vector Under a Pieri In- clusion Removing One Box}

In this section we use the Pieri inclusion $\Phi_{1}$ constructed in Section 3 to give an optimal description of the image under a Pieri inclusion removing one box of a highest weight vector. Given a removal set $X=\left\{x_{1}=\left[b_{1}\right]\left(1, w_{b_{1}}\right)\right\} \subset \lambda$, it is clear by the construction of 1-paths that for all 1-paths on $\lambda$ removing $X,\left(T_{\lambda}\right)_{P}$ is semi-standard.

Define the relation $\sim$ on the set of all 1-paths on $\lambda$ removing $X$ by

$$
P \sim Q \Longleftrightarrow R^{Q} \text { and } R^{P} \text { intersect the same set of rows. }
$$

This clearly defines an equivalence relation. Let

$$
[P]=\{Q: Q \sim P\}
$$


Then for all $Q \in[P]$ we have $(-1)^{Q}=(-1)^{P}$ and $H(Q)=H(P)$, and, when considering the image of a highest weight vector where each entry in a given row is the same,

$$
Y_{Q} \otimes\left(T_{\lambda}\right)_{Q}=Y_{P} \otimes\left(T_{\lambda}\right)_{P}
$$

For distinct $[P]$ and $\left[P^{\prime}\right]$ we have (by construction) that $Y_{P} \otimes\left(T_{\lambda}\right)_{P}$ and $Y_{P^{\prime}} \otimes\left(T_{\lambda}\right)_{P^{\prime}}$ are linearly independent. Thus, $\Phi_{1}\left(T_{\lambda}\right)$ can be written as

$$
\Phi_{1}\left(T_{\lambda}\right)=\sum_{\left[P\left(\sigma_{0}^{A} T\right)\right]} \frac{(-1)^{P\left(\sigma_{0}^{A} T\right)}\left|\left[P\left(\sigma_{0}^{A} T\right)\right]\right|}{H\left(P\left(\sigma_{0}^{A} T\right)\right)} P_{0}\left(T_{\lambda}\right)
$$

where the sum is over all 1-paths $P_{0}$ on $\lambda$ removing $X$ which only hit boxes in the first column of $\lambda$. From the above, the terms in the image of $\Phi_{1}\left(T_{\lambda}\right)$ are linearly independent and do not require straightening, and so this description is optimal. Recall that such an example was computed in Section 1.3. To see the optimal description from this example, take only the first six terms shown.

For a given such 1-path $P_{0}$, we now describe the corresponding term in the image of $T_{\lambda}$. Let $\left\{r_{i}\right\}_{1 \leqslant i \leqslant \mid R^{P_{0} \mid}}$ be the rows in $\lambda$ that $P_{0}$ hits, so that $r_{i}>r_{i+1}$ and $r_{\mid R^{P_{0} \mid}}=\left[b_{1}\right](1)$. Then

$$
\left|\left[P\left(\sigma_{0}^{A} T\right)\right]\right|=\prod_{i=1}^{|P|} \lambda_{r_{i}}
$$

and $\left(T_{\lambda}\right)_{P_{0}} \in \mathbb{S}_{\lambda \backslash X}(V)$ has $\lambda_{1}$ ones in the first row, $\lambda_{2}$ twos in the first row, etc. except for each row $r_{i}, 1 \leqslant i \leqslant\left|R^{P_{0}}\right|$, where the last entry in row $r_{i}$ of $\left(T_{\lambda}\right)_{P_{0}}$ is

$$
\left(\left(T_{\lambda}\right)_{P_{0}}\right)_{\left(r_{i}, \lambda_{r_{i}}\right)}=r_{i+1}
$$

We have implemented this optimal description using Macaulay2, with the output given as a hash table, where one can quickly compute the image of the highest weight for very large examples. Figure 4 shows the timed computation for the image of a highest vector, where the partition is given as the first input of the function oneboxremovalHW and the second input of the function is the row (from the top of the tableau) of the box to be removed.

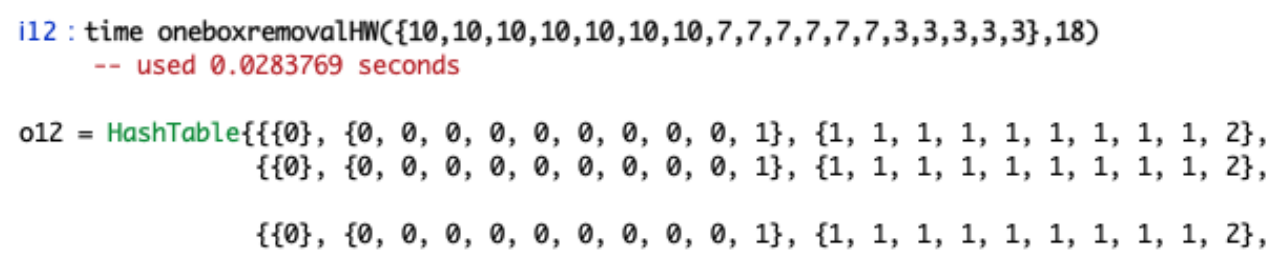

Figure 4: Computing the image of the highest weight for the inclusion $\mathbb{S}_{\left(3^{5}, 7^{6}, 10^{7}\right)}(V) \rightarrow$ $\mathbb{S}_{(1)}(V) \otimes \mathbb{S}_{\left(2^{1}, 3^{4}, 7^{6}, 10^{7}\right)}(V)$. 


\subsection{Comparing Computational Complexity}

We now describe the computational complexity of $\Phi_{1}$ and compare this to the computational complexity of the one box removal Pieri inclusion described by Olver. For a removal set $X=\left\{x_{1}=\left[b_{1}\right]\left(1, w_{b_{1}}\right)\right\}$, let

$$
\widetilde{\Phi}_{1}: \mathbb{S}_{\lambda}(V) \rightarrow V \otimes \mathbb{S}_{\lambda \backslash X}(V)
$$

be the Pieri inclusion given by Olver (see $[14, \S 1.2]$ and $[12, \S 4]$ ).

Theorem 37. Fix a positive integer $N$ and consider partitions $\lambda$ that have at most $N$ blocks. Then our algorithm to compute the image of a highest weight vector under a Pieri inclusion $\Phi_{1}: \mathbb{S}_{\lambda}(V) \hookrightarrow V \otimes \mathbb{S}_{\lambda \backslash X}(V)$ has a worst-case time complexity of $O\left(\ell(\lambda)^{N}\right)$. On the other hand, the algorithm to compute the image of a highest weight vector under a Pieri inclusion $\widetilde{\Phi}_{1}: \mathbb{S}_{\lambda}(V) \hookrightarrow V \otimes \mathbb{S}_{\lambda \backslash X}(V)$ has a worst-case time complexity of $\Omega\left(2^{\ell(\lambda)}\right)$.

Proof. Let $\lambda=\left(w_{1}^{h_{1}}, \ldots, w_{N}^{h_{N}}\right)$. We first consider the time complexity of the algorithm as given by Olver's construction. As before, when considering the image of a highest weight vector we only need to select paths on $\lambda$ removing $X$ that act on the first column of $\lambda$. From the description of the map $\widetilde{\Phi}_{1}$ removing $X$ in $[14, \S 1.2]$, the number of such paths in the computation of $\widetilde{\Phi}_{1}$ is equal to the number of choices of rows in $\lambda$ above row $\left[b_{1}\right](1)$. Thus the complexity of the map $\widetilde{\Phi}_{1}$ acting on a highest weight vector is

$$
2^{h_{b_{1}}-1} \cdot \prod_{i=b_{1}+1}^{N} 2^{h_{i}} \leqslant \frac{1}{2} \cdot \prod_{i=1}^{N} 2^{h_{i}}=\frac{1}{2} \cdot 2^{\sum_{i=1}^{N} h_{i}}=\frac{1}{2} \cdot 2^{\ell(\lambda)} .
$$

In the worst-case when $b_{1}=1$, the inequality is in fact an equality. Furthermore, the paths that act on the first column of $\lambda$ using Olver's algorithm can result in tableaux which are not semi-standard, and so must be straightened. Hence the worst-case complexity of Olver's algorithm is $\Omega\left(2^{\ell(\lambda)}\right)$.

The map $\Phi_{1}$ removing $X$ restricts the choices of rows to those which describe an evacuation route, and hence the number of 1-paths acting on the first column of $\lambda$ in the computation of $\Phi_{1}$ is equal to the number of choices of rows in $\lambda$ above row $\left[b_{1}\right](1)$ made without skipping rows within blocks. It also clear from the definition of 1-paths that the image of a highest weight vector under a 1-path is semi-standard. Thus the complexity of the map $\Phi_{1}$ acting on a highest weight vector is

$$
h_{b_{1}} \cdot \prod_{i=b_{1}+1}^{N}\left(h_{i}+1\right)<\prod_{i=1}^{N}\left(h_{i}+1\right) \leqslant(\ell(\lambda)+1)^{N}=\Theta\left(\ell(\lambda)^{N}\right) .
$$

Remark 38. Similar to the previous theorem, by restricting the maximum possible width of a block in $\lambda$ we get that $\Phi_{1}$ is an exponential speed up of $\widetilde{\Phi}_{1}$ on the image of basis vectors (semi-standard tableaux) in $\mathbb{S}_{\lambda}(V)$. 
This exponential to polynomial speed up can be seen in the computation time for computing Pieri maps in Macaulay2, for which we have implemented the description of $\Phi_{1}$ given in Section 3 within Sam's PieriMaps package [12]. In Figure 5 below we show the timed computations for computing the map

$$
\mathbb{S}_{(8,8,8)}(V) \rightarrow \mathbb{S}_{(1)}(V) \otimes \mathbb{S}_{(8,8,7)}(V)
$$

Using the known algorithm for $\widetilde{\Phi}_{1}$ (as built in to PieriMaps), the process was interrupted after an hour with no output. Using our algorithm implemented in PieriMaps, comuting this map takes only 0.07 seconds.

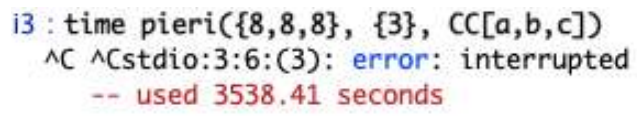

(a) Using the known algorithm.

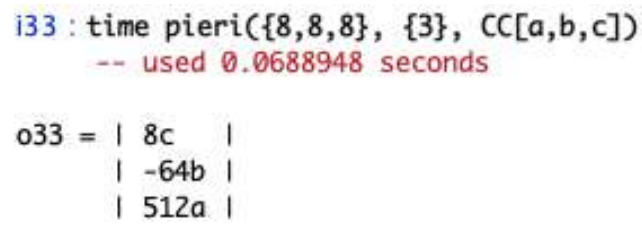

(b) Using our algorithm.

Figure 5: Computing the inclusion $\mathbb{S}_{(8,8,8)}(V) \rightarrow \mathbb{S}_{(1)}(V) \otimes \mathbb{S}_{(8,8,7)}(V)$.

We can also see this exponential speed up for small examples with more than one block. In the figure below we show the computation time for the Pieri inclusion

$$
\mathbb{S}_{\left(1^{9}, 3^{1}\right)}(V) \rightarrow \mathbb{S}_{(1)}(V) \otimes \mathbb{S}_{\left(1^{8}, 3^{1}\right)}(V)
$$

using the known algorithm.

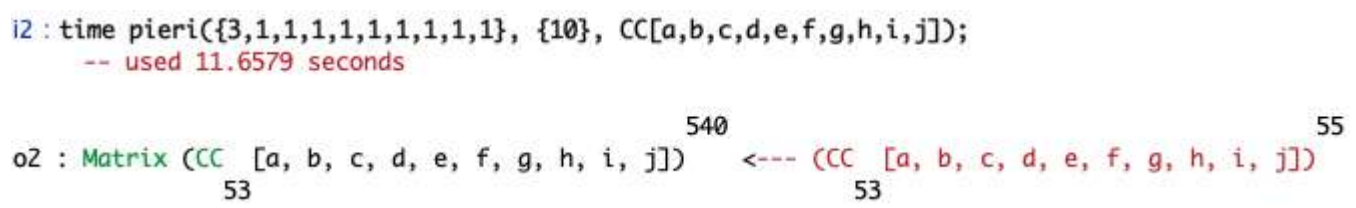

Using the known algorithm this computation takes over eleven seconds, while with the new algorithm this computation (shown below) takes less than two seconds.

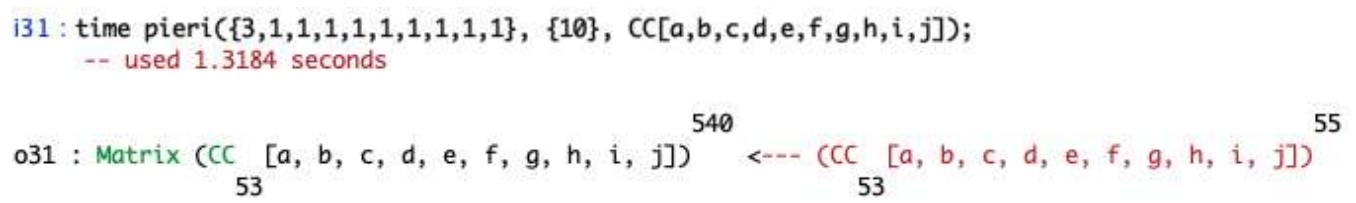

\section{Acknowledgements}

The authors wish to express their gratitude to the referees for their time and helpful suggestions for improving the paper. 


\section{References}

[1] David Eisenbud, Gunnar Fløystad, and Jerzy Weyman. The existence of equivariant pure free resolutions. Annales de l'Institut Fourier, 61(3):905-926, 2011. arXiv:0709.1529v5.

[2] Cameron Farnsworth. Koszul-Young flattenings and symmetric border rank of the determinant. Journal of Algebra, 447:644-676, 2016.

[3] William Fulton and Joe Harris. Representation theory: a first course, volume 129. Springer Science \& Business Media, 2013.

[4] Gunnar Fløystad, Joe Kileel, and Giorgio Ottaviani. The Chow form of the essential variety in computer vision. Journal of Symbolic Computation, 86:97-119, 2018. https://doi.org/10.1016/j.jsc.2017.03.010.

[5] William Fulton. Young tableaux: with applications to representation theory and geometry, volume 35. Cambridge University Press, 1997.

[6] Federico Galetto. Computational methods for orbit closures in a representation with finitely many orbits. Experimental Mathematics, 23(3):310-321, 2014. https://doi.org/10.1080/10586458.2014.904256

[7] Markus Hunziker, John A. Miller, and Mark Sepanski. Minimal Graded Free Resolutions of Modules of Covariants for Classical Groups.

[8] J.M. Landsberg and Giorgio Ottaviani. Equations for secant varieties of Veronese and other varieties. Annali di Matematica Pura ed Applicata, 192(4):569-606, 2013. https://doi.org/10.1007/s10231-011-0238-6

[9] Mihalis Maliakas and Peter J. Olver. Explicit generalized Pieri maps. Journal of Algebra, 148(1):68-85, 1992.

[10] Peter J. Olver. Differential hyperforms I. University of Minnesota Mathematics Report, pages 82-101, 1980.

[11] Piotr Pragacz and Jerzy Weyman. Complexes associated with trace and evaluation. Another approach to Lascoux's resolution. Advances in Mathematics, 57(2):163-207, 1985.

[12] Steven V. Sam. Computing inclusions of Schur modules. Journal of Software for Algebra and Geometry, 1(1):5-10, 2009.

[13] Shlomo Sternberg. Group theory and physics. Cambridge University Press, 1995.

[14] Steven V. Sam and Jerzy Weyman. Pieri resolutions for classical groups. Journal of Algebra, 329(1):222-259, 2011. arXiv:0907.4505v5 